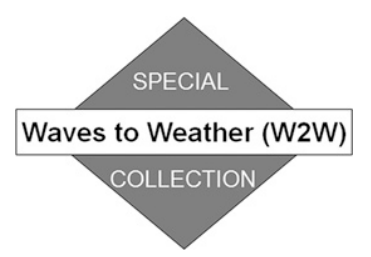

\title{
a Quantitative View on the Processes Governing the Upscale Error Growth up to the Planetary Scale Using a Stochastic Convection Scheme
}

\author{
Marlene Baumgart, PAOlo GHinassi, AND Volkmar Wirth \\ Institut für Physik der Atmosphäre, Johannes Gutenberg-Universität Mainz, Mainz, Germany \\ Tobias SElZ AND GeORge C. CRAIG \\ Meteorologisches Institut, Ludwig-Maximilians-Universität München, Munich, Germany \\ MICHAEL RIEMER \\ Institut für Physik der Atmosphäre, Johannes Gutenberg-Universität Mainz, Mainz, Germany
}

(Manuscript received 16 August 2018, in final form 24 December 2018)

\begin{abstract}
Two diagnostics based on potential vorticity and the envelope of Rossby waves are used to investigate upscale error growth from a dynamical perspective. The diagnostics are applied to several cases of global, real-case ensemble simulations, in which the only difference between the ensemble members lies in the random seed of the stochastic convection scheme. Based on a tendency equation for the enstrophy error, the relative importance of individual processes to enstrophy-error growth near the tropopause is quantified. After the enstrophy error is saturated on the synoptic scale, the envelope diagnostic is used to investigate error growth up to the planetary scale. The diagnostics reveal distinct stages of the error growth: in the first $12 \mathrm{~h}$, error growth is dominated by differences in the convection scheme. Differences in the upper-tropospheric divergent wind then project these diabatic errors into the tropopause region (day 0.5-2). The subsequent error growth (day 2-14.5) is governed by differences in the nonlinear near-tropopause dynamics. A fourth stage of the error growth is found up to 18 days when the envelope diagnostic indicates error growth from the synoptic up to the planetary scale. Previous ideas of the multiscale nature of upscale error growth are confirmed in general. However, a novel interpretation of the governing processes is provided. The insight obtained into the dynamics of upscale error growth may help to design representations of uncertainty in operational forecast models and to identify atmospheric conditions that are intrinsically prone to large error amplification.
\end{abstract}

\section{Introduction}

Weather prediction has improved significantly in the past decades (Bauer et al. 2015). Forecast dropouts, however, do still occur in operational numerical weather prediction models (Rodwell et al. 2013, 2018). Because of the multiscale nature of atmospheric dynamics, there may always be an intrinsic limit of predictability even if model errors and initial-condition errors occur only on

¿ Denotes content that is immediately available upon publication as open access.

Corresponding author: Marlene Baumgart, mbaumga@ uni-mainz.de the smallest resolved scale (Lorenz 1969). Small-scale errors associated with moist processes grow much faster than errors on the synoptic scale and saturate already after about one day (Hohenegger and Schär 2007). Because of this scale dependence, small-scale errors can initiate a multistage sequence of upscale error growth and thereby affect the forecast skill on much larger scales (e.g., Zhang et al. 2003, 2007; Selz and Craig 2015b; Judt 2018).

Based on an idealized moist baroclinic wave simulation, Zhang et al. (2007) derived a conceptual threestage model for upscale error growth. A similar upscale error-growth behavior could also be found in realistic weather events (Selz and Craig 2015b; Judt 2018). In the first stage, errors grow quickly especially in regions of 
moist convection due to convective instability. This growth quickly slows down and saturates on the convective scale with a complete displacement of the individual cells (Zhang et al. 2003, 2007). The following stage is characterized by the transitioning from an unbalanced small-scale error pattern to a balanced largerscale error pattern. Although spreading gravity waves are a prominent feature of this stage (Selz and Craig 2015b), Bierdel et al. $(2017,2018)$ have argued that these have only transient effects that are small in comparison to changes in the net divergence forced by convection. In the last stage, the model suggests that errors grow on the synoptic scale with the background baroclinic instability.

Upscale error growth has often been investigated using error-energy spectra (e.g., Zhang et al. 2007; Selz and Craig 2015b; Judt 2018; Selz 2019). These spectra provide insight into the scale of the error, but do not provide insight into the processes governing the error growth. One diagnostic to provide such insight into the processes governing the error growth is based on potential vorticity (PV; Snyder et al. 2003; Dirren et al. 2003; Davies and Didone 2013; Baumgart et al. 2018). Recently, Baumgart et al. (2018) applied this diagnostic to a case study of an operational forecast. In the presence of the initial-condition and model errors of the investigated forecast of a state-of-the-art operational model, the error growth was dominated by differences in the nonlinear near-tropopause dynamics. This work suggests that these near-tropopause interactions might be more important to synoptic-scale error growth than the direct impact of baroclinic instability. For upscale error growth, one expects that the processes dominating the error growth are more directly related to moist processes than in the operational forecast investigated by Baumgart et al. (2018). To gain deeper insight into the role of moist processes to upscale error growth, we here apply the same diagnostic framework as in Baumgart et al. (2018) to simulations, in which the initial differences stem only from the representation of convection.

On the synoptic scale, forecast errors saturate when troughs and ridges in an upper-level Rossby wave pattern are completely out of phase. Beyond this synopticscale phase saturation at long lead times (beyond 2 weeks), the results of Buizza and Leutbecher (2015) indicate that there is still forecast skill for large-scale fields. To investigate error growth up to the planetary scale, we employ a complementary diagnostic that filters out phase information and identifies the envelope of the upper-level Rossby waves. This diagnostic is based on finite-amplitude local wave activity (LWA) in the primitive-equation, isentropic-coordinates framework following Ghinassi et al. (2018). It has been shown recently that the LWA-based diagnostic is particularly suitable in identifying finite-amplitude wave packets and related nonlinear phenomena such as wave-breaking and blocking (Ghinassi et al. 2018). Using this phasefiltered envelope diagnostic, we are capable to investigate error growth up to the planetary scale and long lead times.

Simulating explicitly the upscale error growth from the convective up to the planetary scale requires convection-permitting simulations on very large, preferably global, domains and for long lead times. Such an approach is computationally extremely costly, but has recently been performed for a single case by Judt (2018). This approach, however, is currently not feasible to be applied to a large number of cases with several ensemble members to ensure the robustness of the results. Using coarser resolution with a deterministic scheme to parameterize convection, upscale error growth turns out to be much slower (Zhang et al. 2003; Selz and Craig 2015a). A way out of this problem was suggested by Selz and Craig (2015a) who showed that a stochastic convection scheme, namely the scheme of Plant and Craig (2008), could generate error amplitudes on meso- and synoptic scales similar to a convection-permitting simulation. The scheme models the individual updrafts in a grid box and draws their magnitude randomly from a distribution. A different realization of the convection can thus be achieved by changing the random seed of the scheme. The scheme thus simulates the outcome of the first error-growth stage and projects it adaptively onto the model grid. For upscale error-growth experiments, this characteristic is a crucial advantage compared to simulations with a deterministic scheme.

Recently, Selz (2019) investigated the intrinsic predictability on larger scales that originate from the short intrinsic predictability of convection using the stochastic convection scheme of Plant and Craig (2008) to represent the first stage of error growth. The coarse resolution allowed for the simulation of 12 cases, regularly distributed over one year, with 5 ensemble members each. In the current work we use this dataset to apply the advanced diagnostics described above to gain more insight into the processes governing the upscale error growth.

This paper is organized as follows: section 2 describes the upscale error-growth simulations and introduces the PV and envelope diagnostics that are used to quantify the error growth from a dynamical, process-based perspective. Section 3 illustrates the results of our diagnostic for one representative case study. The relative importance of the individual processes to upscale error growth is then discussed in section 4. Finally, we provide a summary of our results in section 5 . 


\section{Data and methods}

\section{a. Global simulations with a stochastic convection scheme}

We use the icosahedral nonhydrostatic global forecasting model ICON of the German weather service (Zängl et al. 2015). The model was run with a horizontal resolution corresponding to about $40 \mathrm{~km}$. Convection is parameterized using the stochastic Plant-Craig (PC) convection scheme (Plant and Craig 2008). The PC scheme uses a random value of the convective mass flux, which is drawn from a quasi-equilibrium distribution that uses the ensemble mean mass flux given by a standard CAPE closure. For each ensemble member a different seed is used to generate the random draw, resulting in a different realization of convective variability [see Fig. 1 in Selz (2019) for illustration]. At the initial time, this is the only difference between ensemble members, but as the simulations evolve, differences in the resolved flow will also lead to differences in the closure.

A total of 12 different cases are considered, one for each month from October 2016 to September 2017. These cases are initialized $12 \mathrm{~h}$ before the start of the month, using initial conditions from ECMWF. After a spinup period of $12 \mathrm{~h}$, we create an ensemble of five members by changing the random seed of the PC scheme. Initially, the only difference between these members thus lies in the convective scheme. All simulations are run for a duration of 31 days. Further information on the set up of the simulations are provided in Selz (2019).

For the PV inversion, we use data with a horizontal resolution of $1^{\circ} \times 1^{\circ}$ on pressure levels from 900 to $100 \mathrm{hPa}$ with a grid distance of $50 \mathrm{hPa}$. Data on pressure levels below the ground are created by horizontal linear interpolation from valid neighboring grid points. We use hourly data for the first 10 days and 3-hourly data afterward. For the further analysis of the error growth, data are interpolated from pressure to isentropic levels. Different isentropes are used for each case to account for the seasonal dependence of the tropopause location. For each case, the isentrope is chosen such that the tropopause is located at approximately $45^{\circ} \mathrm{N}$ [see climatology of Liniger and Davies (2004)], resulting in the choice of $315 \mathrm{~K}$ for December, January, and February; $320 \mathrm{~K}$ for November, March, and April; $325 \mathrm{~K}$ for October and May; $330 \mathrm{~K}$ for June and September; and $335 \mathrm{~K}$ for July and August.

\section{b. PV error and tendency equation}

We use Ertel-PV (Ertel 1942) as our key variable. In the framework of the primitive equations and with potential temperature $\theta$ as vertical coordinate, it is defined as

$$
P=\frac{1}{\sigma}\left(\zeta_{\theta}+f\right)
$$

where $\zeta_{\theta}$ is the vertical component of the isentropic relative vorticity, $f$ is the Coriolis parameter, and $\sigma=-g^{-1}(\partial p / \partial \theta)$ is the isentropic layer density with gravitational acceleration $g$ and pressure $p$.

The PV tendency is given by advection and nonconservative PV modification,

$$
\frac{\partial P}{\partial t}=-\mathbf{v} \cdot \nabla_{\theta} P+N+\text { res. }
$$

where $N=-\dot{\theta}(\partial P / \partial \theta)+P(\partial \dot{\theta} / \partial \theta)+(1 / \sigma) \mathbf{k} \cdot(\nabla \times \dot{\mathbf{v}}) \mathrm{de}-$ scribes the nonconservative $\mathrm{PV}$ modification due to diabatic heating and nonconservative momentum change. The heating rates $\dot{\theta}$ and horizontal wind tendencies $\dot{\mathbf{v}}$ are approximated by centered differences of accumulated tendencies ${ }^{1}$ from the parameterization schemes (convection, microphysics, long- and shortwave radiation, turbulence, subgrid-scale orography, and gravity wave drag). The residual (res.) includes the influence of those nonconservative processes that cannot be quantified with the available data as, e.g., the contribution of numerical diffusion, and intrinsic numerical errors due to the discretization and interpolation of the data.

Our focus is on the difference in PV between the individual ensemble members, $\Delta P$, defined as

$$
\Delta P=P_{n}-P_{m},
$$

where $P_{n}$ and $P_{m}$ denote the PV of two different ensemble members $n$ and $m$, respectively. Our ICON ensemble contains five members. It is thus possible to define the PV difference for 10 different member pairs: member 2 - member 1 , member 3 - member 1 , member 3 - member $2, \ldots 2$ In the following, we use the terminology "error" for the difference between two members although, strictly speaking, none of the members describes the true evolution.

To have a positive definite metric, we consider the (potential) enstrophy error, $(\Delta P)^{2} / 2$. The mean enstrophy

\footnotetext{
${ }^{1}$ Data are saved with an hourly resolution for the first 10 days and with a 3-hourly resolution afterward (see section $2 a$ ), so the heating rates and horizontal wind tendencies are approximated by 2- and 6-h-centered differences, respectively.

${ }^{2}$ Note that for the further analysis of the error amplification it is not necessary to investigate the other possible combination of two members (e.g., one does not need to investigate member 1 member 2 in addition to member $2-$ member 1 ) as enstrophy and LWA are positive definite variables.
} 
error $\mathscr{P}$ is then defined as the spatial average of the enstrophy error over the midlatitudes of the Northern Hemisphere $\left(30^{\circ}-80^{\circ} \mathrm{N}\right)$,

$$
\mathscr{P}=\frac{1}{A} \int_{A} \frac{(\Delta P)^{2}}{2} d A,
$$

where $A$ is the area of integration, $d A=a^{2} \cos \phi d \lambda d \phi$ is the area element in spherical coordinates, $a$ denotes the radius of Earth, $\lambda$ is longitude, and $\phi$ is latitude. $\mathscr{P}$ is then averaged over the 12 cases and 10 member pairs within each case, which allows us to obtain reasonably robust statistics (a sample of 120), yielding $\overline{\mathscr{P}}$. The statistical uncertainty of $\mathscr{\mathscr { P }}$ associated with the month-tomonth variability is estimated as the standard error for the 12 cases [i.e., $\sigma(\overline{\mathscr{P}})=\sigma / \sqrt{12}$, where $\sigma$ denotes the standard deviation of the 12 cases].

To further investigate the enstrophy-error evolution, we follow the derivation of Baumgart et al. (2018) and derive a tendency equation for $\Delta P$

$$
\frac{\partial \Delta P}{\partial t}=-\Delta \mathbf{v} \cdot \nabla_{\theta} \bar{P}-\overline{\mathbf{v}} \cdot \nabla_{\theta} \Delta P+\Delta N+\text { res. }
$$

where

$$
\begin{aligned}
\Delta N= & -\Delta \dot{\theta} \frac{\partial \bar{P}}{\partial \theta}-\overline{\dot{\theta}} \frac{\partial \Delta P}{\partial \theta}+\Delta P \frac{\partial \dot{\dot{\theta}}}{\partial \theta}+\bar{P} \frac{\partial \Delta \dot{\theta}}{\partial \theta} \\
& +\frac{1}{\bar{\sigma}} \mathbf{k} \cdot(\nabla \times \Delta \dot{\mathbf{v}})+\frac{1}{\Delta \sigma} \mathbf{k} \cdot(\nabla \times \overline{\dot{\mathbf{v}}})
\end{aligned}
$$

denotes the tendency of $\Delta P$ due to nonconservative processes. Variables with a $\Delta$ denote the difference between the two members, while variables with an overbar denote the mean of the two members. We further extend the tendency for the PV error to a tendency for the enstrophy error:

$$
\begin{aligned}
\frac{\partial}{\partial t} \frac{(\Delta P)^{2}}{2}= & -\Delta P \Delta \mathbf{v} \cdot \nabla_{\theta} \bar{P}-\nabla_{\theta} \cdot\left[\frac{(\Delta P)^{2}}{2} \overline{\mathbf{v}}\right] \\
& +\frac{(\Delta P)^{2}}{2} \nabla_{\theta} \cdot \overline{\mathbf{v}}+\Delta P \Delta N+\text { res. }
\end{aligned}
$$

We partition the advective part of the error tendency based on the PV perspective for midlatitude dynamics (Hoskins et al. 1985) as in Teubler and Riemer (2016) and Baumgart et al. (2018). We analyze the contributions to error growth near the tropopause by decomposing the horizontal velocity at that near-tropopause level. For that purpose, we first apply a Helmholtz partitioning to separate the divergent flow from the nondivergent flow (Lynch 1989). The nondivergent flow is then further partitioned into the flow associated with upper- and lowerlevel PV anomalies, respectively, by using piecewise PV inversion (PPVI; Davis and Emanuel 1991; Davis 1992).
Anomalies are defined as deviations from a background state, which for each case is defined as the temporal mean over the 31 days of simulation time averaged over the 5 members. Piecewise PV inversion is performed between $25^{\circ}-85^{\circ} \mathrm{N}$ and $850-150 \mathrm{hPa}$. Vertical boundary conditions are specified as potential temperature anomalies at 875 and $125 \mathrm{hPa}$. Here $600 \mathrm{hPa}$ is used as the separation level between upper- and lower-level PV anomalies as PV errors are, in general, only small at this midlevel [Fig. 2 in Baumgart et al. (2018)]. The partitioning includes uncertainties due to the harmonic flow component, the horizontal boundary conditions, and the nonlinearities of the piecewise PV inversion. In general, these uncertainties are small and do not affect the physical interpretation of the results.

The influence of upper-level PV anomalies (by PV advection due to the associated winds) on the neartropopause evolution is here interpreted as the contribution from near-tropopause dynamics $\left(\mathbf{v}_{\mathrm{nTP}}\right)$. As shown by Baumgart et al. (2018), this contribution to neartropopause error growth is mostly related to differences in the nonlinear dynamics. ${ }^{3}$ The influence of lower-level $\mathrm{PV}$ and $\theta$ anomalies on the near-tropopause evolution is here interpreted as the contribution from troposphericdeep interaction $\left(\mathbf{v}_{\mathrm{TPd}}\right)$. This contribution describes the vertical interaction between upper and lower levels and includes the basic mechanism for baroclinic instability (Eady 1949; Hoskins et al. 1985; Davis and Emanuel 1991; Heifetz et al. 2004). The influence of uppertropospheric divergence $\left(\mathbf{v}_{\text {div }}\right)$ can be associated with balanced dynamics and diabatic processes [as one would obtain from an omega equation, see, e.g., chapter 6.4 in Holton and Hakim (2012)]. A strong divergence contribution is often associated with latent heat release below (Davis et al. 1993; Riemer et al. 2014; Quinting and Jones 2016). In summary, our flow partitioning results in

$$
\mathbf{v} \approx \mathbf{v}_{\mathrm{nTP}}+\mathbf{v}_{\mathrm{TPd}}+\mathbf{v}_{\mathrm{div}} .
$$

We insert this flow partitioning in Eq. (7) and spatially integrate over the midlatitude Northern Hemisphere $\left(30^{\circ}-80^{\circ} \mathrm{N}\right)$. Thereby, we can quantify the relative importance of near-tropopause dynamics [term 1 on the righthand side of Eq. (9)], tropospheric-deep interaction (term 2), upper-tropospheric divergence (terms 3 and 4), and nonconservative processes (term 5) to enstrophy-error growth:

\footnotetext{
${ }^{3}$ In Baumgart et al. (2018), the partitioning into the contribution of linear and nonlinear dynamics, respectively, was based on the difference between the full near-tropopause tendency and its linearized part where the background state of the PPVI (30-day temporal mean) was used as reference state for the linearization (see their section $4 b$ ).
} 


$$
\begin{aligned}
\frac{d \mathscr{P}}{d t}= & \frac{1}{A} \int_{A} \frac{\partial}{\partial t} \frac{(\Delta P)^{2}}{2} d A=\frac{1}{A}\left[-\int_{A} \Delta P \Delta \mathbf{v}_{\mathrm{nTP}} \cdot \nabla_{\theta} \bar{P} d A-\int_{A} \Delta P \Delta \mathbf{v}_{\mathrm{TPd}} \cdot \nabla_{\theta} \bar{P} d A\right. \\
& \left.-\int_{A} \Delta P \Delta \mathbf{v}_{\mathrm{div}} \cdot \nabla_{\theta} \bar{P} d A+\int_{A} \frac{(\Delta P)^{2}}{2} \nabla_{\theta} \cdot \overline{\mathbf{v}_{\mathrm{div}}} d A+\int_{A} \Delta P \Delta N d A\right]+ \text { bnd. }+ \text { res }
\end{aligned}
$$

The boundary term, bnd. $=-(1 / A) \oint_{S}\left[(\Delta P)^{2} / 2\right] \overline{\mathbf{v}} \cdot \mathbf{n} d S$, describes the boundary effect due to errors being advected into or out of the integration domain. Because of the choice of the integration domain (hemispheric integration from $30^{\circ}$ to $80^{\circ} \mathrm{N}$ ), this boundary contribution is in general very small and will not be considered hereafter.

In addition to the enstrophy-error tendency, we consider the growth rate associated with the individual processes:

$$
\alpha_{\text {process }}=\left.\frac{1}{\mathscr{P}}\left(\frac{d \mathscr{P}}{d t}\right)\right|_{\text {process }} .
$$

In contrast to the enstrophy-error-tendency diagnostic [Eq. (9)], which provides a quantitative metric for the absolute error growth, this growth-rate diagnostic provides a quantitative metric for the error growth relative to the existing error. By combining both diagnostic, we thus have a quantitative metric for both the absolute and relative error growth.

As for the mean enstrophy error $(\overline{\mathscr{P}})$, the enstrophyerror tendency [Eq. (9)] and growth rates [Eq. (10)] associated with the individual processes are averaged over the 12 cases and the 10 member pairs within each case to obtain reasonably robust statistics. ${ }^{4}$

\section{c. Rossby wave envelope error}

The Rossby wave envelope is obtained by, first, quantifying Rossby "waviness" through local finiteamplitude wave activity (LWA) and then extracting the envelope through a spatial filter. In our work we decided to use the envelope of LWA instead of the more established envelope of the meridional wind (Zimin et al. 2003). While both yield very similar results during the linear stage of a Rossby wave, the former was shown to be more appropriate in the case of finiteamplitude waves (Ghinassi et al. 2018).

Our LWA diagnostic is defined in the framework of the primitive equations with potential temperature $\theta$ as

\footnotetext{
${ }^{4}$ If a PV inversion at one time step did not converge for a specific case and member (about $5 \%$ of all inversions), the average of the near-tropopause and tropospheric-deep tendency shown in Figs. 7 and 8 is taken only over the converged cases and member pairs. Because of the small number of not-converged time steps, we still expect our diagnostic to be reasonably robust.
}

vertical coordinate. Details can be found in Ghinassi et al. (2018). Broadly speaking, LWA is a positive definite quantity related to the displacement of PV contours from zonal symmetry. Consider a specific isentropic layer at a specific time. For any given latitude $\phi$ one defines an associated PV contour $Q(\phi)$ through the relation

$$
\iint_{P \geq Q} \sigma d A=\iint_{\phi^{\prime} \geq \phi} \sigma d A
$$

where $d A$ denotes surface integration on the considered isentrope, the integration on the left-hand side extends over those areas where $\mathrm{PV}$ is larger than $Q$, and the integration on the right-hand side extends poleward of the considered latitude $\phi$. LWA is then defined as

$$
\begin{aligned}
\operatorname{LWA}(\lambda, \phi)= & \frac{1}{\cos \phi}\left\{\int_{l_{S}}\left[P\left(\lambda, \phi^{\prime}\right)-Q(\phi)\right] \sigma a \cos \phi^{\prime} d \phi^{\prime}\right. \\
& \left.+\int_{l_{N}}\left[Q(\phi)-P\left(\lambda, \phi^{\prime}\right)\right] \sigma a \cos \phi^{\prime} d \phi^{\prime}\right\},
\end{aligned}
$$

where $\phi^{\prime}$ denotes the integration variable in the meridional direction, and $l_{S}$ and $l_{N}$ are the arcs along the meridian at any given $\lambda$ satisfying the following conditions:

$$
\begin{aligned}
& l_{S}: P \geq Q, \phi^{\prime} \leq \phi, \\
& l_{N}: P \leq Q, \phi^{\prime} \geq \phi,
\end{aligned}
$$

[for illustration see Fig. 1 in Ghinassi et al. (2018)]. LWA has units of meters per second and quantifies the strength of Rossby waves in terms of their pseudomomentum. The zonal average of LWA exactly recovers the finiteamplitude wave activity of Nakamura and Solomon (2011). LWA is Eulerian in longitude and partly Lagrangian in latitude. This characteristic has to be kept in mind when comparing LWA-based diagnostics with our earlier-defined PV-based diagnostics, since the latter is fully Eulerian. In addition, the LWA-based diagnostic is nonlocal in the sense that (possibly remote) nonconservative processes affect the relation between $\phi$ and $Q$. This effect renders the relation $Q(\phi)$ dependent on time; however, this time dependence turns out to be small in the sense that $Q(\phi)$ varies by less than $10 \%$ within a 
one-month period and, by consequence, the difference between $P$ and $Q$ in the expression for LWA is dominated by variations in $P$. Regarding our ensembles, it is appropriate to define a common $Q(\phi)$ relation for all members, and this is achieved by using the corresponding relation at initial time where all ensemble members are identical. This guarantees that LWA at one latitude $\phi$ is computed with respect to the same PV contour $Q(\phi)$ for each ensemble member. For computational efficiency, we use a spatial resolution of $2^{\circ} \times 2^{\circ}$ for the computation of LWA.

LWA as defined above still contains the phase information of the wave. We now go on to filter out this phase dependence through a convolution with a Hann window in the zonal direction, which results in the wave envelope $E$. The filter is allowed to depend on longitude and its width is estimated through a wavelet analysis as described in Ghinassi et al. (2018).

In our analysis we consider the difference $\Delta E$ in the envelope between two ensemble members $n$ and $m$,

$$
\Delta E=E_{n}-E_{m} .
$$

Note that $\Delta E$ (in contrast to $E$ ) is a signed variable and includes both amplitude and position errors of the envelope.

As for the enstrophy error, we consider the squared envelope error, $(\Delta E)^{2} / 2$, which is a positive definite quantity. From this, the mean squared envelope error is calculated by a spatial average yielding

$$
\mathscr{E}=\frac{1}{A} \int_{A} \frac{(\Delta E)^{2}}{2} d A
$$

where the integration area $A$ extends over the midlatitudes of the Northern Hemisphere $\left(20^{\circ}-80^{\circ} \mathrm{N}\right)$. Finally, $\mathscr{E}$ is averaged over the 12 cases and 10 member pairs within each case yielding $\overline{\mathscr{E}}$. The statistical uncertainty of $\overline{\mathscr{E}}$ associated with the month-to-month variability is estimated as the standard error for the 12 cases [i.e., $\sigma(\overline{\mathscr{E}})=\sigma / \sqrt{12}]$.

\section{Illustrative case study}

The October ensemble is used to illustrate the spatial patterns of our error-growth diagnostics. This section starts with a discussion of the differences between the first two members. Recall that the difference between two ensemble members is here referred to as error. The contributions from the individual processes to error growth are then discussed in terms of their spatial pattern. Finally, the error growth in the twomember ensemble is compared to that in the fivemember ensemble.

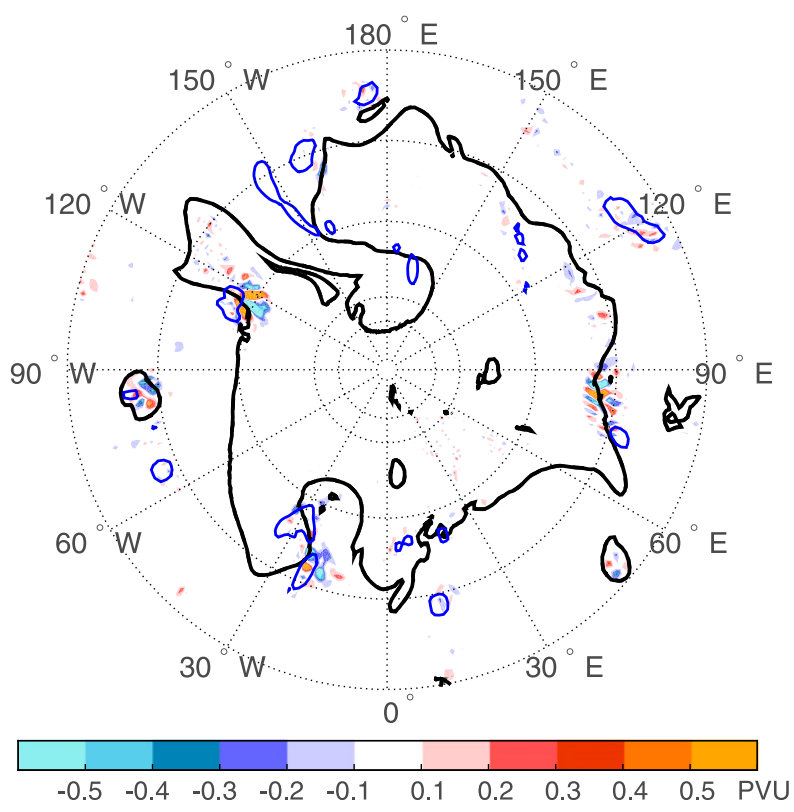

FIG. 1. PV error derived from the first two members $\left(\Delta P=P_{2}-P_{1}\right)$ of the October ensemble at day 1 . Black contours denote the dynamical tropopause of member 1 (solid) and member 2 (dashed). The blue contours depict the $1 \mathrm{~mm} \mathrm{~h}^{-1}$ precipitation averaged over member 1 and 2 .

\section{a. Evolution of error patterns}

The PV and envelope error ( $\Delta P$ and $\Delta E)$ derived from the first two members of the October ensemble at selected time steps are shown in Figs. 1-3. At day 1, the PV error occurs near the grid scale and exhibits considerable values only in localized regions (Fig. 1). These regions are spatially correlated with regions of precipitation. Between day 1 and 5 (Figs. 1 and 2a), the PV error amplifies by an order of magnitude (note the different scales of the color bars) and starts to develop spatially more coherent patterns. Differences in the position of the dynamical tropopause become apparent by day 5 . During these first five days the envelope error is mostly related to amplitude errors, but overall only of very small magnitude (not shown).

Between day 5 and 10, the PV error further amplifies (Figs. 2a and 2b). Importantly, the rather localized PV errors change to a larger-scale and more coherent error pattern that maximizes in regions where the tropopause is highly distorted. The PV error now projects more strongly on the envelope error (Fig. 3g), which shows a dipole error around $120^{\circ}-150^{\circ} \mathrm{W}$, suggesting that the error consists in a shift of the envelope. The second envelope-error maximum is a monopole located around $30^{\circ}-60^{\circ} \mathrm{E}$, suggesting instead an error in the amplitude of the envelope. 

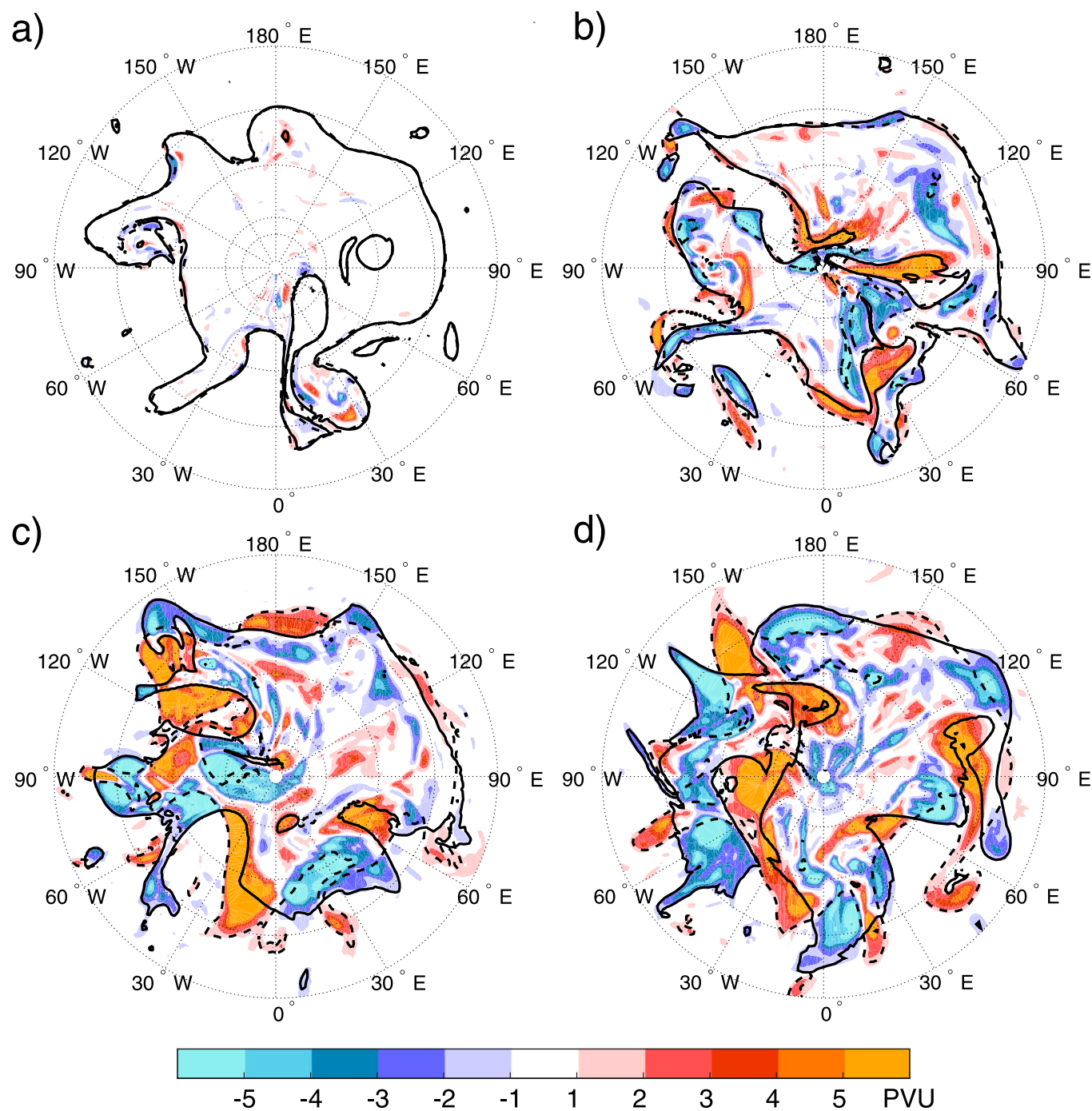

FIG. 2. PV error derived from the first two members $\left(\Delta P=P_{2}-P_{1}\right)$ of the October ensemble at (a) day 5 , (b) day 10, (c) day 15, and (d) day 20. Black contours denote the dynamical tropopause of member 1 (solid) and member 2 (dashed).

At day 15, the PV error exhibits approximately the same scale and magnitude as the troughs and ridges within the Rossby wave pattern (Fig. 2c). At the same time, the envelope error affects an extended part of the midlatitudes of the Western Hemisphere, with two large negative errors over the northeast Pacific and North Atlantic sector and a positive error in between (Fig. 3h). The region with a more zonally oriented tropopause $\left(70^{\circ}-140^{\circ} \mathrm{E}\right)$ shows only small errors both in PV and the envelope.

Between day 15 and 20, the PV error amplification is less pronounced than previously (Figs. $2 \mathrm{c}$ and $2 \mathrm{~d}$ ), indicating that the PV error starts to saturate. The Rossby wave pattern in the Western Hemisphere seems to be completely decorrelated. This decorrelation appears as a large error in the envelope diagnostic, mostly in the form of an amplitude error (Fig. 3i). At the same time, more pronounced PV errors start to occur in the Eastern Hemisphere. These errors also become apparent as small errors in the envelope diagnostic.

\section{b. Error tendencies associated with individual processes}

In terms of PV, we can go one step further and relate the error evolution to individual processes. The spatial patterns of the individual contributions to the enstrophy-error tendency [Eq. (9)] are illustrated in Fig. 4 . The time steps and regions are chosen such that the respective process makes a dominant contribution to the error growth. 

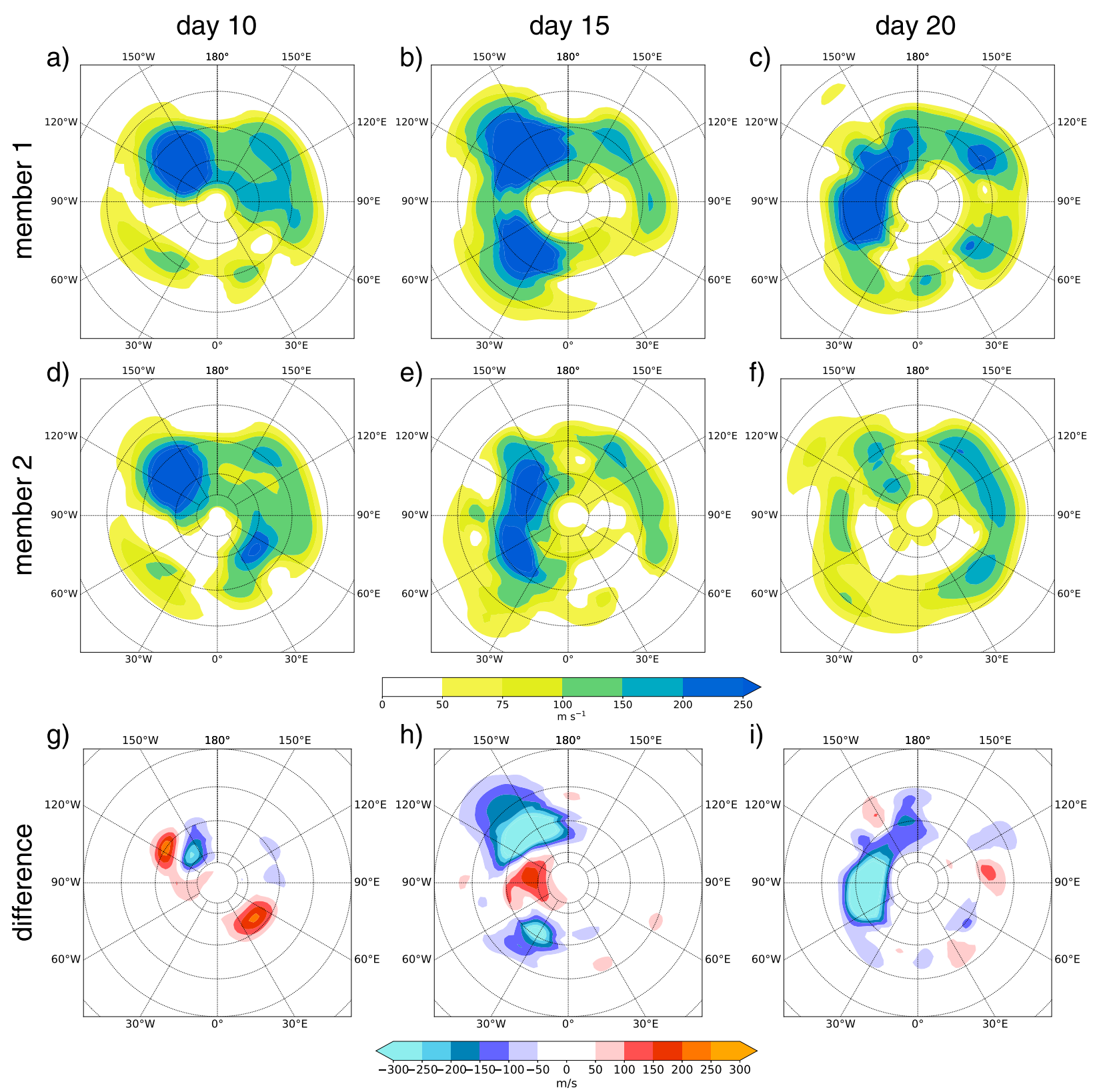

FIG. 3. Rossby wave envelope $E$ from member 1 of the October ensemble at (a) day 10, (b) day 15, and (c) day 20. (d)-(f) As in (a)-(c), but for member 2. (g)-(i) As in (a)-(c), but for the envelope difference $\Delta E$.

The nonconservative tendency at $18 \mathrm{~h}$ (Fig. $4 \mathrm{a}$ ) occurs near the grid scale in localized regions of precipitation. In these regions, there is also a significant precipitation error (Fig. 4b). A partitioning of the nonconservative tendency into the contributions from the individual parameterization schemes (see below in section 4b, Fig. 7b) reveals that the contribution of the convection scheme dominates at this time, suggesting that differences in the latent heating induced by the convection scheme govern the error growth at that time.
The divergence tendency at day 1.5 (Fig. 4c) leads to error amplification along the dynamical tropopause, particularly in the two ridges and the cutoff. A large divergence tendency is found in the vicinity of cyclones where also the vertical velocity exhibits a pronounced error (Fig. 4d). At this early lead time, the verticalvelocity error and the associated divergence error tendency are still rather small-scale and mainly within an envelope of expected mesoscale ascent, as in the warm conveyor belt region around $20^{\circ} \mathrm{W}$. This observation 
a) day 0.75 . nonconservative error tendency at $325 \mathrm{~K}$

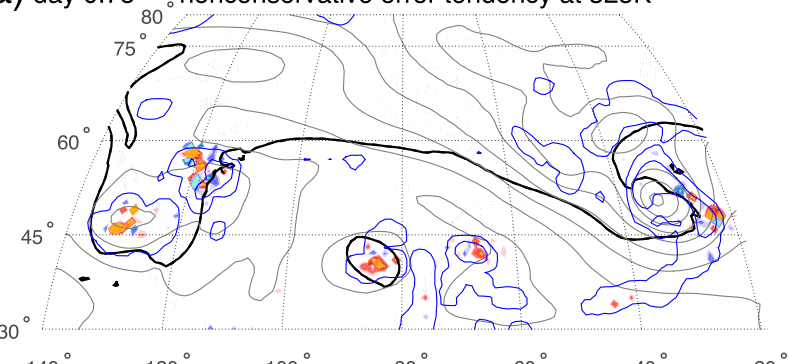

b) day 0.75 precipitation error

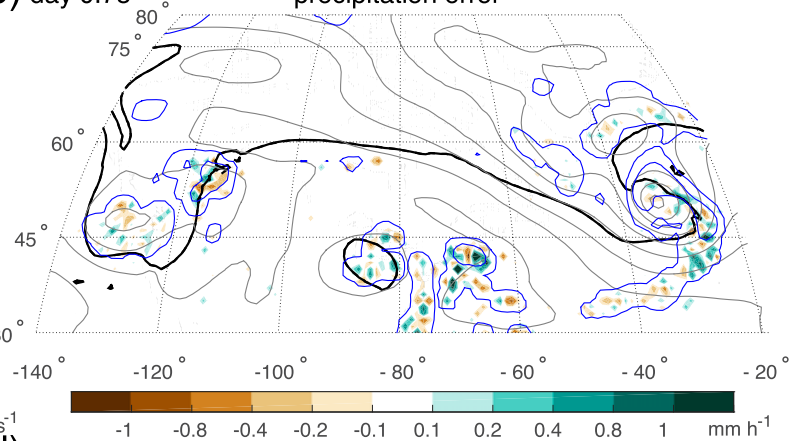

d) day $1.5^{-1}$

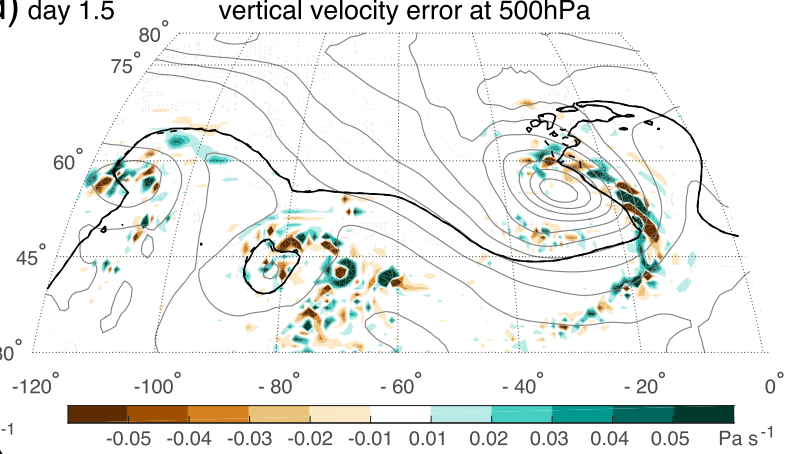
$20^{\circ}$
C) day $1.5 \quad$ divergent error tendency at $325 \mathrm{~K}$

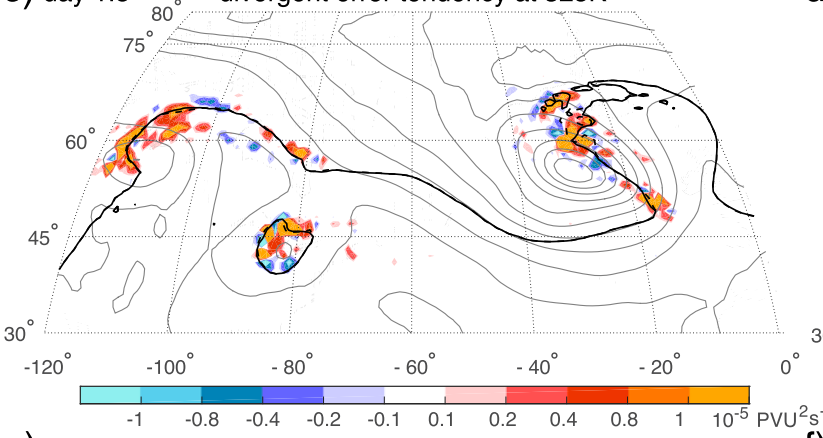

e) day $4 \quad 80^{\circ}$ near-tropopause error tendency at $325 \mathrm{~K}$

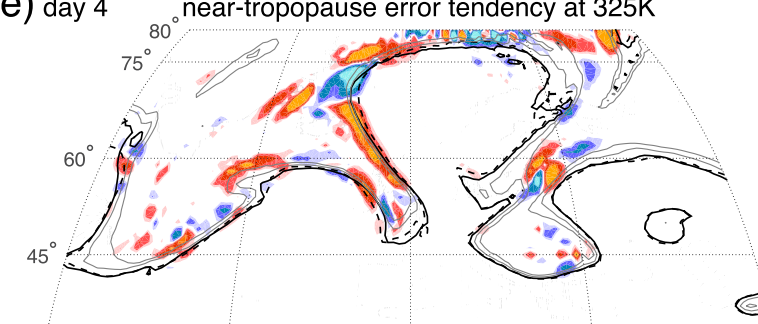

$30^{\circ}$ $-60^{\circ}$

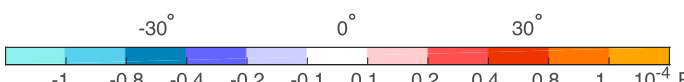

g) day 22 tropospheric-deep error tendency at $325 \mathrm{~K}$

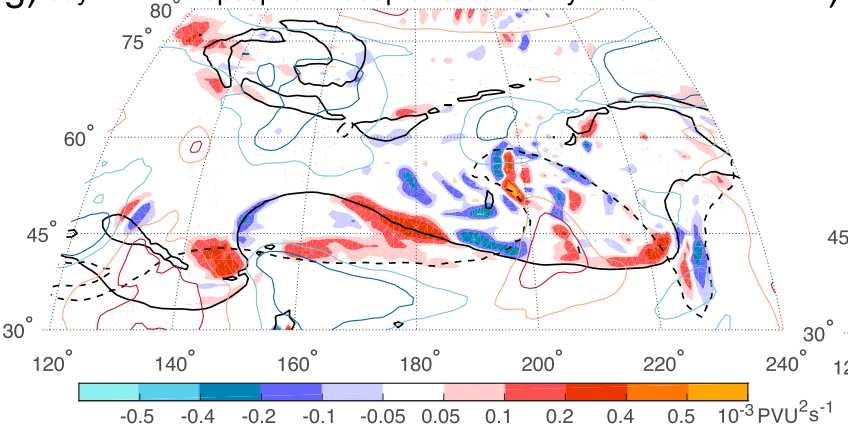

f) day 4
$\mathrm{PV}$ error at $325 \mathrm{~K}$

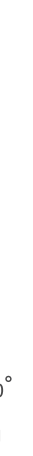

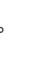

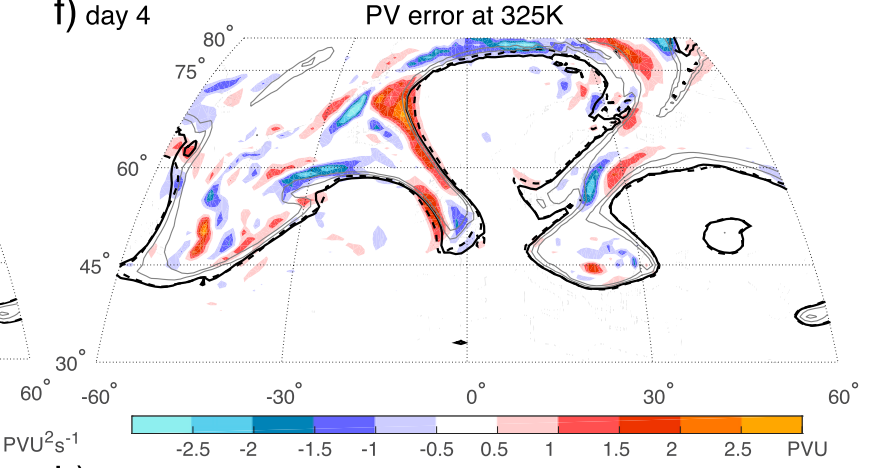

h) day $2280^{\circ}$ potential temperature error at $875 \mathrm{hPa}$

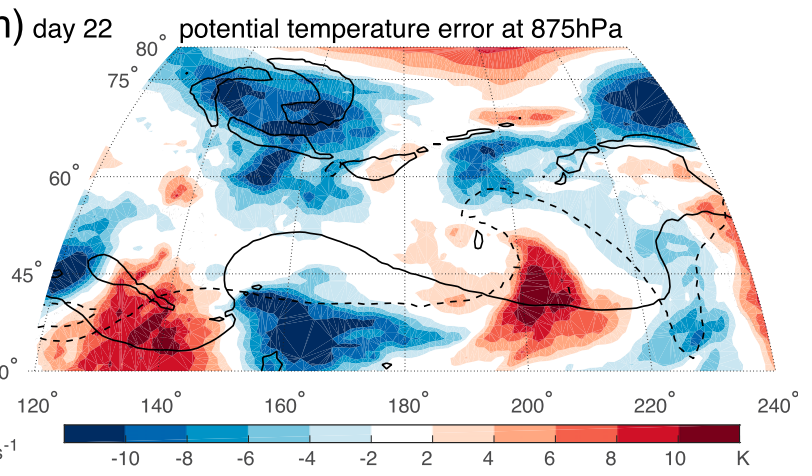

FIG. 4. (left) Individual contributions to the enstrophy-error tendency [Eq. (9)] and (right) together with the error field it is mostly related to. The time and region of the panels are chosen such that the individual processes make a dominating contribution and thus differ for the individual panels. Thick black contours denote the dynamical tropopause at $325 \mathrm{~K}$ of member 1 (solid) and member 2 (dashed). Thin contours depict the mean over member 1 and 2 of (a)-(d) geopotential at $850 \mathrm{hPa}$ every $500 \mathrm{~m}^{2} \mathrm{~s}^{-2}$ (smoothed over 3 grid points) in gray, (a),(b) precipitation at 0.1 and $1 \mathrm{~mm} \mathrm{~h}^{-1}$ (smoothed over 3 grid points) in blue, and (e),(f) PV at 3 and 4 PVU in gray. Contours in (g) denote the potential temperature error at $875 \mathrm{hPa}$ at -4 and $-8 \mathrm{~K}$ (blue) and at 4 and $8 \mathrm{~K}$ (red). 
suggests that the differences in latent heating induced by the convection scheme (see previous paragraph) result in differences in the vertical motion within existing features of ascent, which are projected into the tropopause region by upper-tropospheric divergence. This pattern is in contrast to later lead times, when differences in vertical velocity are rather associated with a displacement of the features themselves (not shown).

The near-tropopause tendency at day 4 (Fig. 4e) maximizes along the dynamical tropopause on the flanks of the narrow trough and the high-amplitude ridge. Large error amplification is found in regions where the PV error aligns with a strong PV gradient (Fig. 4f), consistent with the observation of Snyder et al. (2003) that the PV error maximizes in regions where the PV gradient of the reference flow is large. The regions of nonlinear Rossby wave behavior are associated with large near-tropopause error growth, consistent with the large importance of nonlinear Rossby wave dynamics to the error growth found by Baumgart et al. (2018).

The tropospheric-deep tendency at day 22 (Fig. 4g) leads to error amplification in regions of large-scale PV errors (as can be inferred by the respective 2-PVU contours; 1 PVU $=10^{-6} \mathrm{~K} \mathrm{~kg}^{-1} \mathrm{~m}^{2} \mathrm{~s}^{-1}$ ). In these regions, potential temperature errors near the surface have a similar scale and are in vicinity of the neartropopause PV errors (Fig. 4h). The Eady (1949) model for baroclinic instability suggests that such a configuration is a necessary condition for tropospheric-deep error amplification (Baumgart et al. 2018). Amplification occurs when the lower-level potential temperature errors and upper-level PV errors have a phase shift between 0 and $\pi$. The tropospheric-deep tendency thus quantifies error growth due to differences in the baroclinic growth. Before day 15 when the error has a smaller horizontal scale than the troughs and ridges, the tropospheric-deep error amplification is small (not shown). This small importance of tropospheric-deep interaction to the error growth during the first 15 days is plausible as the vertical penetration of temperature anomalies, and thereby also of temperature errors, depends on their horizontal scale (Eady 1949; Baumgart et al. 2018).

\section{c. Ensemble of all five members}

In the following subsection, we consider the differences within the whole five-member ensemble to investigate how representative the previous error-growth discussion of the two-member ensemble is for the fivemember ensemble. For that purpose, Fig. 5 shows the 2-PVU contour (dynamical tropopause) of all five members for the same time steps as in Fig. 2.

At day 1 (not shown), no difference in the location of the dynamical tropopause is apparent between the individual members, indicating that the error occurs near the grid scale and does not yet affect the synoptic scale. At day 5 (Fig. 5a), differences in the tropopause location of the individual members start to occur. These differences increase significantly until day 10 (Fig. 5b) and an increased spread of the 2-PVU contours is evident in regions of a highly distorted tropopause. Individual troughs and ridges show both phase and amplitude errors, but are still located at similar positions. By day 15, the troughs and ridges are almost completely decorrelated (Fig. 5c), indicating that the PV error becomes saturated on the scale of individual troughs and ridges. The region with a rather zonal tropopause pattern $\left(70^{\circ}-140^{\circ} \mathrm{E}\right)$, in contrast, stands out as a region with only small spread of the tropopause location. At day 20 (Fig. 5d), the differences in the ensemble are apparently not related to individual troughs and ridges any longer.

Compared to the error evolution in the two-member ensemble, the differences between all five members evolve in a similar way both in terms of the typical scale, typical magnitude, and typical location of the error: errors generated near the grid scale start to affect the synoptic scale at around day 5 and reach the scale of the Rossby wave troughs and ridges at around day 15 . This change in the scale of the error is also associated with a strong increase in the magnitude of the error. Regions with a distorted tropopause are associated with large error growth, whereas regions with a more zonal tropopause exhibit only weak error growth. We conclude that the error-growth characteristics discussed in the previous subsections for the two-member ensemble are representative for the five-member ensemble.

\section{Quantitative view on the processes governing the error growth}

To quantify the relative importance of the processes governing the error growth, we consider all 12 cases including all pairwise combinations of the 5-member ensembles. The large number of cases and member pairs provides a large enough sample for statistics to be reasonably robust, namely a sample of 120 . This section starts with a discussion of time series of the mean enstrophy error and mean squared envelope error. Afterward, the contributions of individual processes to error growth are quantified. Spatial maps of the squared envelope error are then discussed to identify "error hot spots." Finally, our results are compared with the conceptual three-stage error growth model of Zhang et al. (2007).

\section{a. Time series of error evolution}

Time series of the mean enstrophy error $(\overline{\mathscr{P}})$ and mean squared envelope error $(\overline{\mathscr{P}})$ over the midlatitudes 

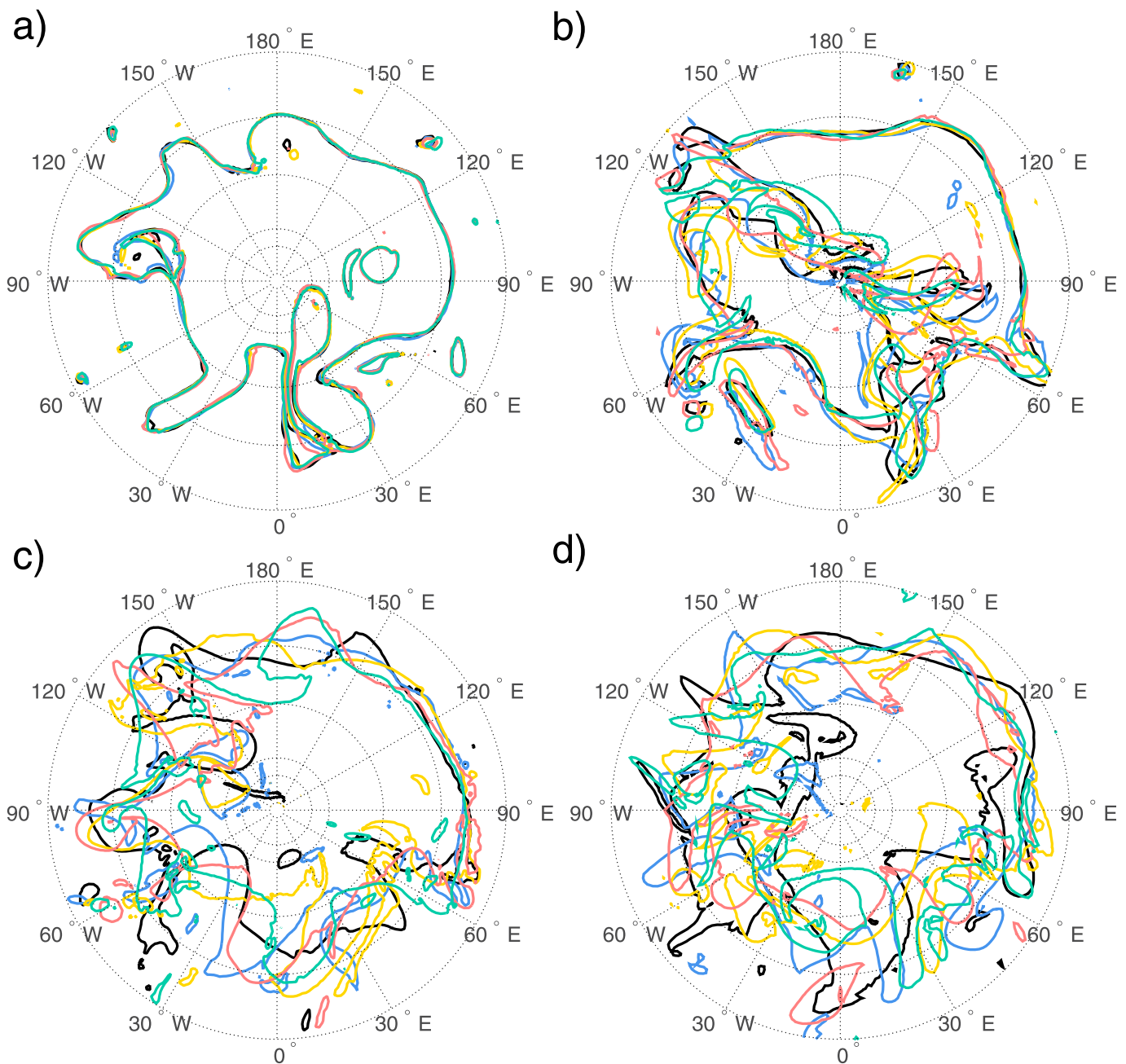

d)

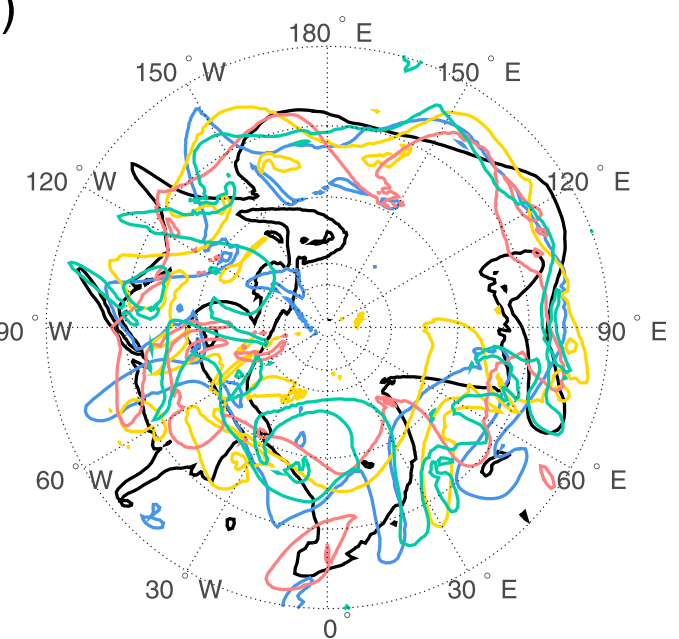

FIG. 5. Dynamical tropopause of member 1 (black contour), member 2 (blue contour), member 3 (yellow contour), member 4 (red contour), and member 5 (green contour) at (a) day 5, (b) day 10, (c) day 15, and (d) day 20.

of the Northern Hemisphere are shown in Fig. 6 together with the (statistical) standard error for the cases.

Both the enstrophy and the envelope diagnostic show the typical form of an error-growth function (e.g., Selz 2019): The initially very fast error growth seems to follow a power-law behavior in the first two days (as seen on a $\log -\log$ plot, not shown), which is followed by quasiexponential growth until day 6-7. Afterward, error growth slows down significantly, until the error eventually reaches saturation after a certain time.

Two main differences exist between the enstrophy and the envelope diagnostic. First, the envelope diagnostic shows larger differences between the 12 cases as measured by the standard error. Second and more importantly, the envelope error saturates at a later time than the enstrophy error.

To quantify the different saturation levels in the enstrophy and envelope diagnostic, we calculate the predictability time $\tau$ in the two diagnostics, according to Selz (2019). For that purpose, the temporal development of $\overline{\mathscr{P}}$ and $\overline{\mathscr{E}}$ after day 7 is fitted to the following function with the fitting parameters $a$ and $b$ :

$$
\overline{\mathscr{H}}=\overline{\mathscr{H}}\left(t_{0}\right) \exp \left(a\left\{1-\exp \left[-b\left(t-t_{0}\right)\right]\right\}\right),
$$

where $t_{0}$ is set to 7 days as in Selz (2019) and $\overline{\mathscr{H}}$ denotes either $\overline{\mathscr{P}}$ or $\overline{\mathscr{E}}$. From Fig. 6 it is evident that Eq. (17) provides a very good fit for the temporal development of $\overline{\mathscr{P}}$ and $\overline{\mathscr{E}}$, respectively.

The predictability time is defined by the time when $80 \%$ of the error magnitude at $t \rightarrow \infty$ is reached, that means

$$
\overline{\mathscr{H}}(\tau)=0.8 \overline{\mathscr{H}}\left(t_{\infty}\right)=0.8 \overline{\mathscr{H}}\left(t_{0}\right) \exp (a) .
$$

With this definition, we derive a predictability time of about 14.5 days for the enstrophy diagnostic and of 

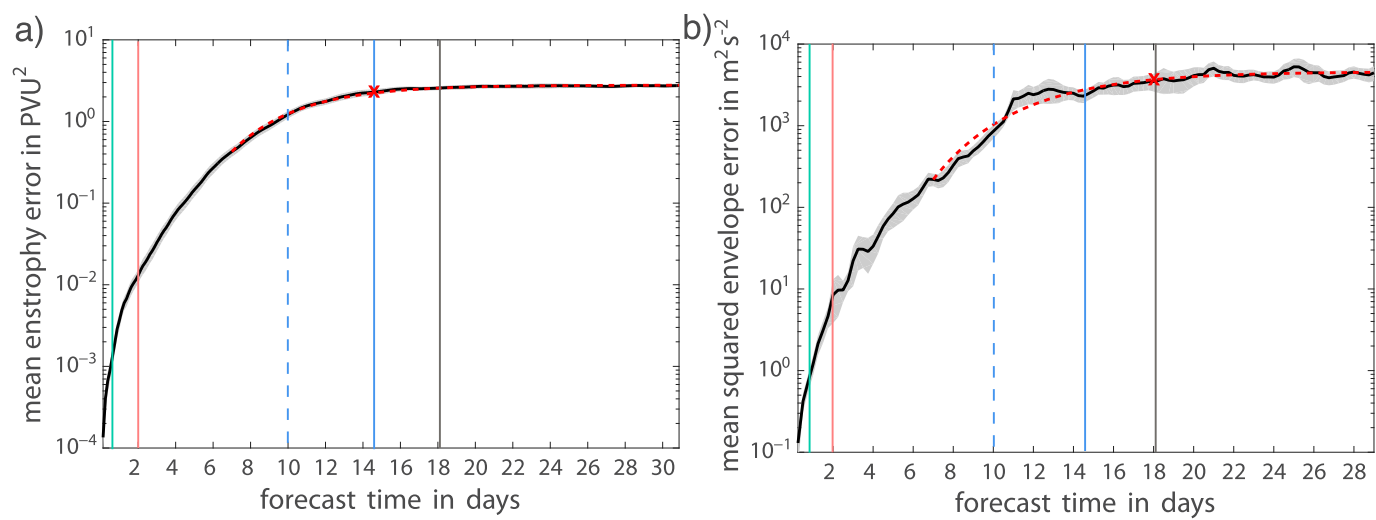

FIG. 6. Time series of (a) mean enstrophy error $(\overline{\mathscr{P}})$, and (b) mean squared envelope error $(\overline{\mathscr{E}})$ over the midlatitudes of the Northern Hemisphere; $\mathscr{P}$ and $\mathscr{E}$ are averaged over the 12 cases and 10 member pairs within each case. These averages are shown as thick black lines, while the shading next to the lines denotes the (statistical) standard error for the 12 cases. The red dashed line shows the fit of the temporal development of the mean enstrophy error and mean squared envelope error to Eq. (17) and the red cross indicates the predictability time [as measured by Eq. (18)] of the diagnostics. For later references, vertical solid lines denote the end of the individual stages, while the dashed blue line denotes the time of maximal near-tropopause error growth (see Figs. 7 and 8).

about 18 days for the envelope diagnostic. For difference kinetic energy, Selz (2019) derived a predictability time of 17 days, which lies in between the result for the enstrophy and envelope diagnostic. This intermediate time can be explained by the fact that the enstrophy spectrum has larger weight on small scales than kinetic energy, while small scales are filtered out in the envelope diagnostic.

Based on Fig. 6, we could thus quantitatively derive a time lag of 3.5 days between the enstrophy diagnostic maximizing on the synoptic scale and the envelope diagnostic maximizing on the planetary scale. The predictability of individual ridges and troughs in a Rossby wave pattern is thus about 3.5 days shorter than the predictability of the wave-pattern envelope.

\section{b. Relative error growth}

For the early stage of the error evolution, we consider the growth rate associated with the individual processes [Eq. (10)] to quantify the error growth relative to the existing error. Time series of these growth rates, averaged over all cases and member pairs, are shown in Fig. 7 together with the (statistical) standard error.

During the first $12 \mathrm{~h}$, the by far largest growth rate is associated with the nonconservative processes (Fig. 7a). A further partitioning of this growth rate (Fig. 7b) indicates that it is mostly related to the convection scheme. The growth rate associated with the convection scheme is at the beginning about $7 \times 10^{-5} \mathrm{~s}^{-1}$ and then sharply decreases by one order of magnitude within the first $12 \mathrm{~h}$. During the first $12 \mathrm{~h}$, error growth is thus dominated by the latent-heating differences that were induced by the convection scheme. In the first $12 \mathrm{~h}$, the longwave radiation and microphysics scheme are associated with error growth, while the turbulence scheme is associated with error decay. These growth rates, however, are much smaller than the growth rate associated with convection in the first $12 \mathrm{~h}$. After about $18 \mathrm{~h}$, when the growth rate associated with the convective scheme is small, these parameterized nonconservative processes become of larger importance to the error growth than the parameterized convection.

A substantial positive residual exists in our budget equation for the enstrophy error in the first $12 \mathrm{~h}$. This residual translates to a growth rate of similar magnitude as the growth rate associated with the convection scheme (not shown) and can be attributed to the fact that our diagnostic measures the growth of existing errors, but not the generation of new errors, which makes a dominating contribution in the very beginning of the simulations. We do not see any indication that this residual associated with the generation of new errors compromises our interpretation of the relative importance of the processes governing the error growth.

The growth rate associated with upper-tropospheric divergence exhibits large values already at the beginning of the simulations and becomes the dominating errorgrowth contribution after about $12 \mathrm{~h}$ when the growth rate associated with the convection scheme has rapidly decreased. The divergence error growth during this stage is, in general, associated with pronounced vertical velocity errors embedded within regions of mesoscale ascent in the vicinity of cyclones (see section 3b, Figs. 4c and $4 \mathrm{~d}$ ). Upper-tropospheric divergence thus provides 

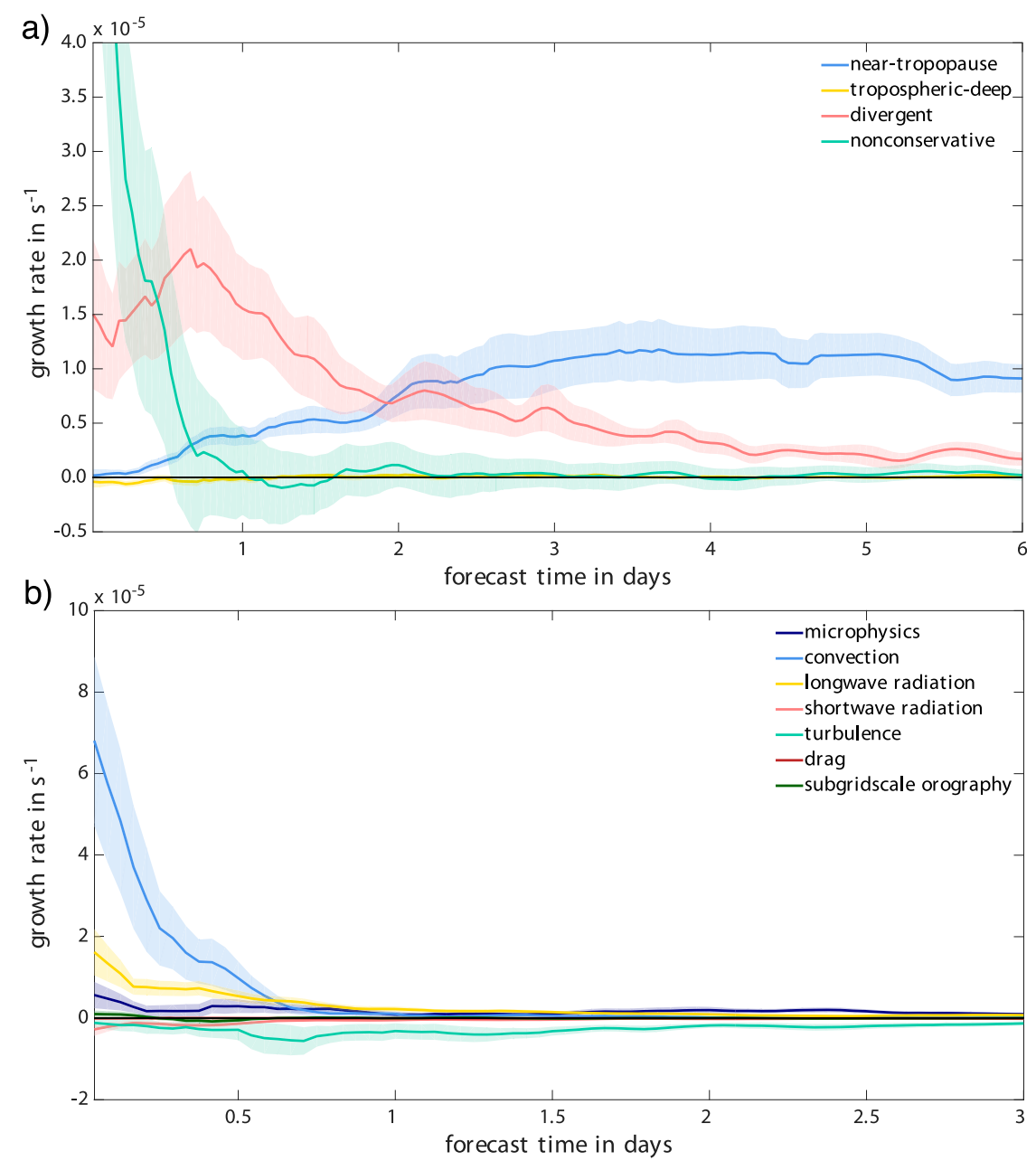

FIG. 7. (a) Growth rate of enstrophy error associated with the individual processes as indicated by Eq. (10), and (b) further partitioning of the nonconservative contribution into the contributions of the individual parameterization schemes. Results are averaged over the 12 cases and the 10 member pairs within each case. These averages are shown as a thick black line, while the shading next to the line denotes the (statistical) standard error for the 12 cases. The time series are smoothed by a running mean over 5 time steps.

the dominating error-growth mechanism to project the latent-heating differences induced by the convection scheme into the tropopause region.

After about 2 days, the growth rate associated with near-tropopause dynamics becomes larger than the growth rate associated with upper-tropospheric divergence, indicating that differences in the nonlinear Rossby wave dynamics near the tropopause become more important to the error growth than differences in the moist processes and upper-tropospheric divergence. The growth rate associated with near-tropopause dynamics is about $1 \times 10^{-5} \mathrm{~s}^{-1}$ and thereby one order of magnitude smaller than the growth rate associated with the convection scheme at the beginning of the simulations. Hohenegger and Schär (2007) found a similar magnitude for error-growth rate on the synoptic scale and also assumed that balanced dynamics are governing the synoptic-scale error growth.

\section{c. Absolute error growth}

For the later stage of the error growth, we consider the individual contributions to the enstrophy-error tendency [Eq. (9)] to quantify the error growth in an absolute sense. Time series of the individual error-tendency contributions, averaged over all cases and member pairs, are shown in Fig. 8 together with the (statistical) standard error.

From the growth-rate discussion (see section $4 b$ ), it is clear that near-tropopause dynamics makes the largest contribution to error growth after about 2 days. 
a)

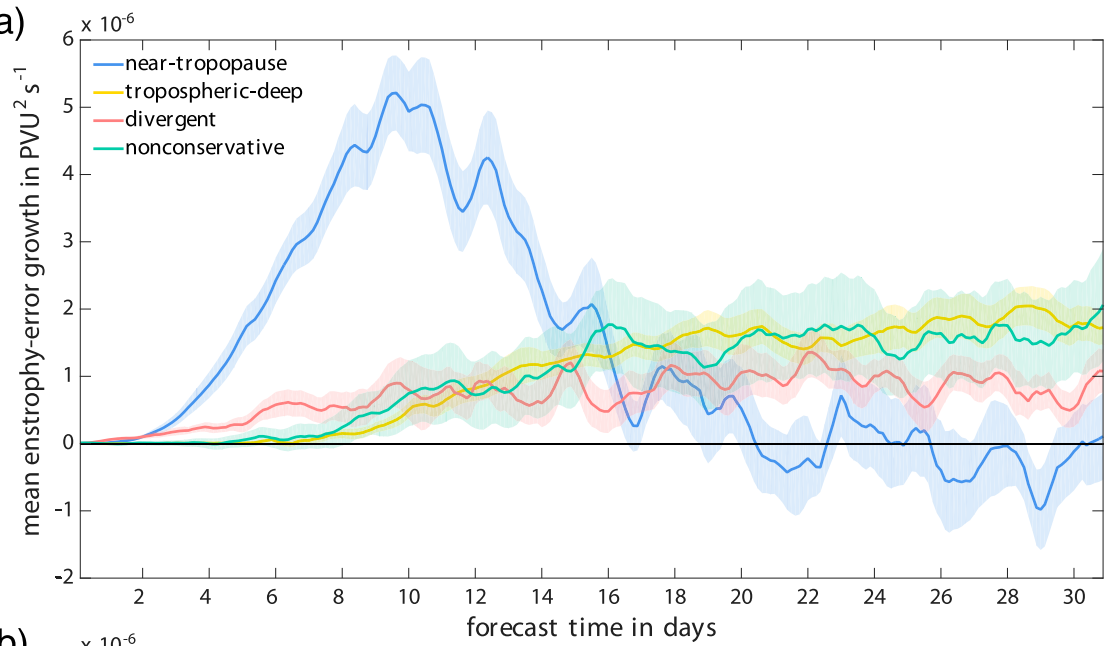

b)

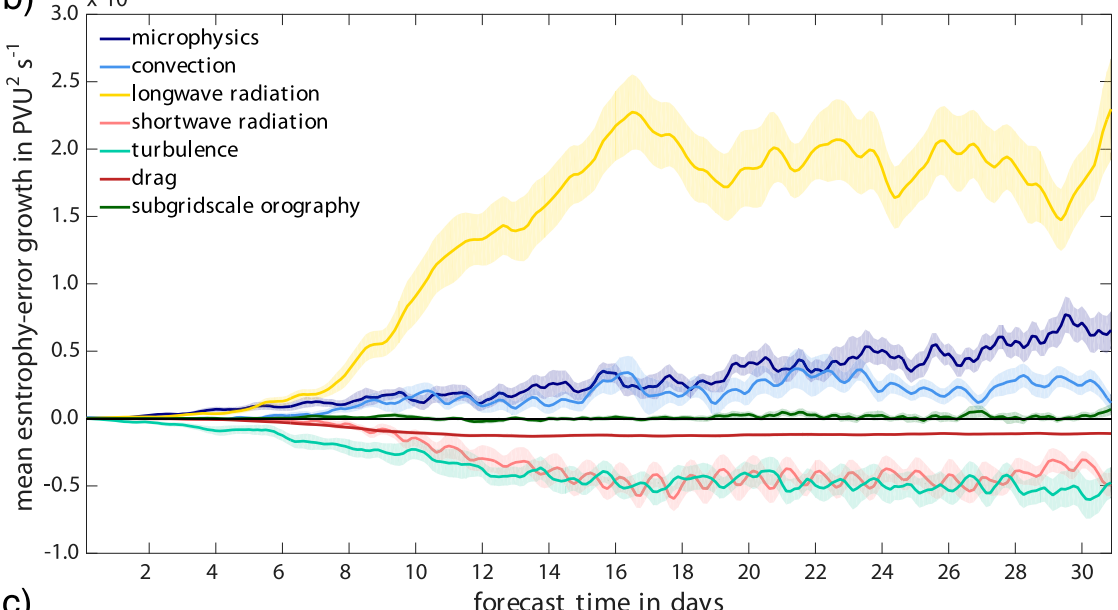

c)

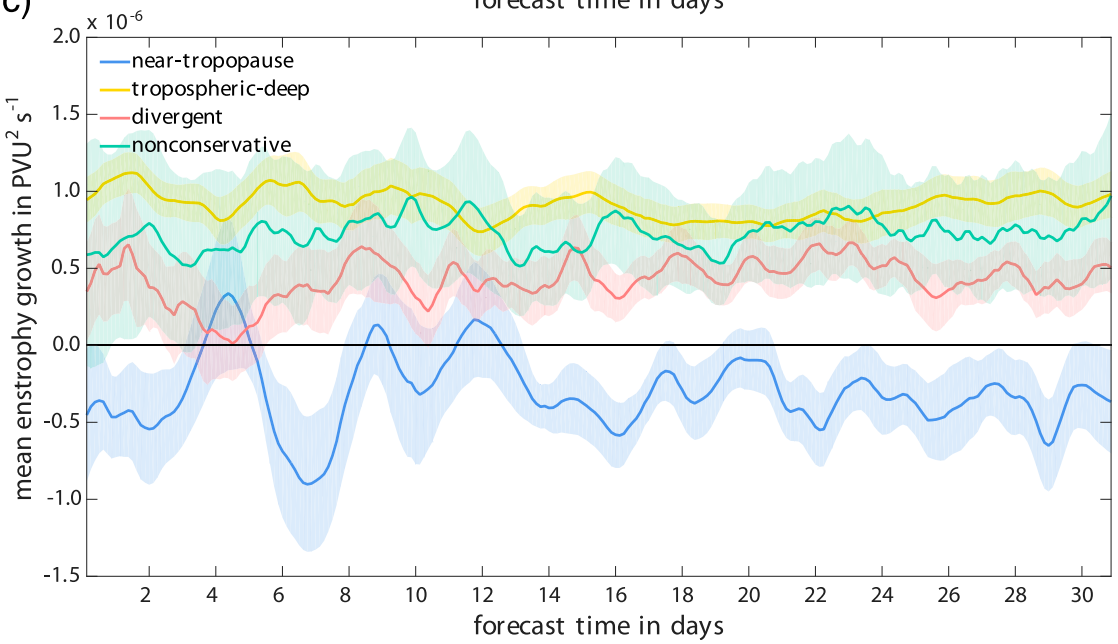

FIG. 8. (a) Partitioning of the mean enstrophy-error tendency into the contributions from the individual processes, (b) partitioning of the mean nonconservative enstrophy-error tendency into the contributions from the individual parameterization schemes, and (c) partitioning of the mean enstrophy tendency into the contributions from the individual processes. The individual error-tendency contributions in (a) and (b) are averaged over the 12 cases and the 10 member pairs within each case, while the individual enstrophy-tendency contributions in (c) are averaged over the 12 cases and the 5 members within each case. These averages are shown as thick lines, while the shading next to the lines denotes the (statistical) standard error for the 12 cases. The time series are smoothed by a running mean over 5 time steps. 
Until day 10, the contribution of near-tropopause dynamics to the error growth is strongly increasing with lead time (Fig. 8a). In the following 7 days, it is decreasing with time, until it is eventually fluctuating around zero after about 17 days. After about 15 days, the contribution of near-tropopause dynamics is no longer the largest error-growth contribution. Interestingly, 15 days is also about the time when the predictability time of enstrophy is reached (see section 4a, Fig. 6). Large error amplification by near-tropopause dynamics thus occurs until the error becomes saturated on the synoptic scale of individual troughs and ridges.

After about 15 days, the nonconservative, troposphericdeep, and divergence contributions become more important to the error growth than the near-tropopause contribution. The large contribution of nonconservative processes is mostly related to the longwave radiation scheme (Fig. 8b). The longwave radiative PV tendency correlates with the PV anomalies of troughs and ridges. Once these anomalies start to decorrelate, this correlation leads to a systematic radiative error amplification. Tropospheric-deep error amplification occurs due to differences in the release of baroclinic instability when the PV and $\theta$ errors near the tropopause and near the surface, respectively, establish a favorable configuration for baroclinic growth (see section 3b, Figs. $4 \mathrm{~g}$ and $4 \mathrm{~h}$ ). The divergence error amplification can be related to a misrepresentation of surface cyclones and their associated divergent outflow in warm conveyor belts (not shown).

To gain insight into the processes governing underlying Rossby wave evolution, we evaluate an analog tendency equation for the enstrophy evolution as derived in Baumgart et al. (2018) (their section 4c). The enstrophy evolution in our simulation (averaged over all cases and members, Fig. 8c) is governed by enstrophy amplification by the nonconservative, divergence, and tropospheric-deep contribution, while the neartropopause contribution is weakly negative. After the enstrophy error reaches the scale of individual Rossby wave troughs and ridges (around day 15), the processes governing the error evolution are thus very similar to the processes governing the underlying Rossby wave evolution. The magnitude of the individual error-tendency contributions is about twice as large as the magnitude of the respective enstrophy-tendency contributions, which is consistent with the double penalty effect of phase errors during error saturation. In this stage, the processes governing the error growth thus resemble the climatological signal of the underlying Rossby wave evolution.

From Fig. $8 \mathrm{c}$ it is clear that the sum of all contributions to enstrophy growth is positive, suggesting that enstrophy would grow during the whole simulation time. Such continuous growth, however, is not physically meaningful as the Rossby wave amplitude is, on average, not amplifying with time. Rossby wave patterns amplify by (moist) baroclinic instability and dissipate at a similar rate by (barotropic) dissipation on the small scales due to the downscale cascade of enstrophy. In numerical weather prediction models, this dissipation appears as numerical diffusion, which is an additional enstrophy sink that cannot be quantified with the available data. The residual in the enstrophy tendency is about $-2 \times$ $10^{-6} \mathrm{PVU}^{2} \mathrm{~s}^{-1}$ and almost constant over time (not shown). A negative residual exists also in the enstrophyerror tendency. This residual increases in magnitude from day 2 to 15 , until it reaches an almost constant value of about $-4 \times 10^{-6} \mathrm{PVU}^{2} \mathrm{~s}^{-1}$ after day 15 (not shown). This value is about twice as large as the residual in the enstrophy tendency, consistent with the observed double penalty effect of phase errors during error saturation. The important role of dissipation was also discussed in previous studies (Snyder et al. 2003; Zhang et al. 2007; Saffin et al. 2016, 2017; Baumgart et al. 2018). For instance, Zhang et al. (2007) quantified the contribution of horizontal and vertical diffusion to their difference kinetic energy budget and found that this kind of dissipation is of similar relative importance to the error evolution as the residual observed in the current study.

\section{d. Spatial distribution of the envelope error}

The spatial distribution of the squared envelope error, $(\Delta E)^{2} / 2$, at day $5,10,15$, and 20 is shown in Fig. 9. At day 5 , the envelope error appears still very localized and small in magnitude. At day 10 (Fig. 9b), two distinct error regions are found around $120^{\circ}-90^{\circ} \mathrm{W}$ and around $30^{\circ} \mathrm{W}$. By day 15 (Fig. 9c), large error amplification occurs in the Western Hemisphere: an extended region of large error is found around $150^{\circ}-110^{\circ} \mathrm{W}$ and secondary maxima are found around $180^{\circ}$ and over an "error belt" extending from North America toward Europe. Between day 15 and 20 (Figs. 9c and 9d), error growth continues further both in magnitude and in its spatial extension. At day 20 (Fig. 9d), the error affects almost the whole mid- to high latitudes of the Northern Hemisphere. At this stage, the predictability time in the envelope diagnostic is already reached (see section $4 \mathrm{a}$, Fig. 6b).

From Fig. 9 it is evident that the envelope error tends to appear and amplify more in the western portion of the Northern Hemisphere, whereas in the eastern portion its magnitude is lower and its spatial extent more confined. This difference may be due to the fact that in our cases the Rossby wave amplitude was, in general, weaker in the eastern than in the western Northern Hemisphere. 

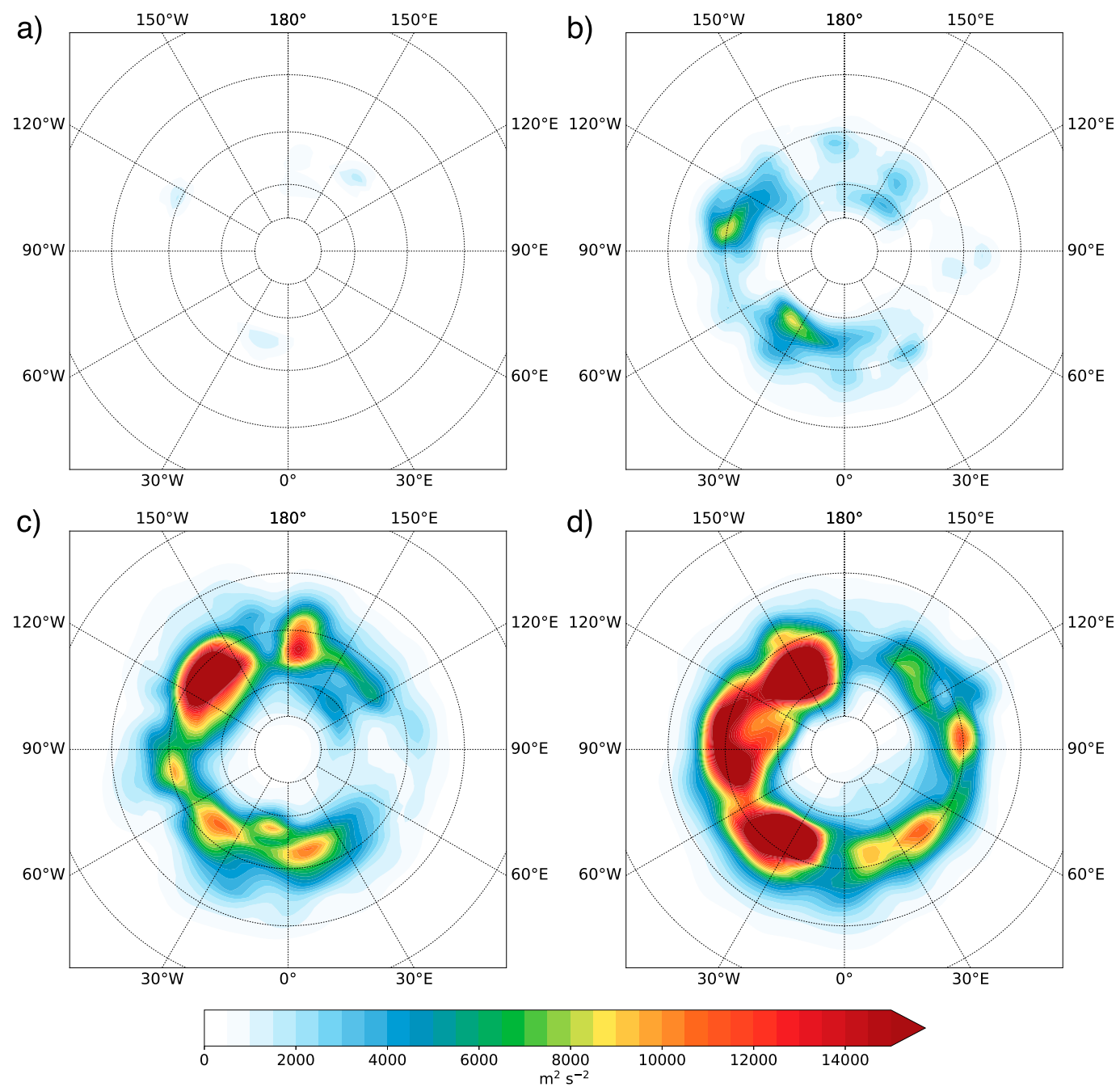

FIG. 9. Squared envelope error $(\Delta E)^{2} / 2$ averaged over the 12 cases and 10 member pairs within each case at (a) day 5, (b) day 10, (c) day 15, and (d) day 20.

\section{e. Comparison with the conceptual three-stage error growth model}

Our quantitative results from the PV- and envelopebased diagnostic reveal that upscale error growth can be divided into distinct stages based on the processes governing the error growth (as indicated by the vertical lines in Fig. 6). In the first stage (0-12h), differences in the convection scheme dominate the error growth, while upper-tropospheric divergence dominates in the second stage ( $0.5-2$ days). In the third stage (2-14.5 days), near-tropopause dynamics dominates the error growth. A fourth stage is found between 14.5 and 18 days, which is characterized by the planetary-scale error growth in the envelope diagnostic. In the following, we will compare these four stages with the conceptual three-stage error growth model derived by Zhang et al. (2007).
Stage 1 of the conceptual model is characterized by error growth on the convective scale in regions of moist convection (Zhang et al. 2007; Selz and Craig 2015b; Judt 2018). The stochastic convective scheme in our study represents explicitly resulting variability by reshuffling of the individual clouds. ${ }^{5}$ The net effect of this stage on the grid scale in the first few hours of the simulations is dominated by differences in the diabatic PV modification from the convection scheme.

In the conceptual model, stage 2 is related to the transitioning toward error growth on the synoptic scale (Zhang et al. 2007; Selz and Craig 2015b). In our

\footnotetext{
${ }^{5}$ Note that this is different from a deterministic convection scheme where the individual updrafts are not simulated, but only the cloud distribution mean.
} 
simulations, the latent-heating differences induced by the convection scheme project on differences in the vertical motion and the associated upper-tropospheric divergent PV advection that appear within regions of mesoscale ascent in the vicinity of cyclones. The transition is in our simulation thus characterized by the divergent flow making a dominant contribution to the error growth. Thereby, upper-tropospheric divergence provides an effective mechanism to project errors from moist processes into the tropopause region.

The third stage in the conceptual model is related to error growth by the background baroclinic instability (Zhang et al. 2007). Although our diagnostic shows that the underlying Rossby wave evolution during this stage is governed by (moist) baroclinic amplification (Fig. 8c), we find that the error growth is governed by differences in the nonlinear near-tropopause dynamics (Fig. 8a). Our diagnostic thereby provides a new interpretation of the processes governing the error growth during this stage and emphasize the importance to distinguish the processes governing the underlying Rossby wave evolution from the processes governing the error growth [see also section 4c in Baumgart et al. (2018)].

We find a fourth stage of the error growth between about 14.5 and 18 days when the predictability time is reached for the enstrophy error, but not for the envelope error. This fourth stage could not be identified by Zhang et al. (2007) and Selz and Craig (2015b) because these studies did not have a long enough lead time to identify this stage. ${ }^{6}$ The enstrophy error maximizes when the phase of the individual troughs and ridges in the Rossby wave pattern becomes decorrelated. The envelope diagnostic, instead, filters phase information and its error can continue to grow beyond the saturation of phase errors. The fourth stage is thus characterized by error growth from the synoptic scale of individual Rossby wave troughs and ridges up to the planetary scale.

In summary, previous ideas of a multiscale behavior of upscale error growth are confirmed by our diagnostics. Furthermore, our results provide a novel interpretation of the governing processes and suggest a fourth stage of upscale error growth.

\section{Summary}

This study provides a quantitative view on the processes governing upscale error growth. Employing the

\footnotetext{
${ }^{6}$ In addition, there are differences in the experimental setup [e.g., the $f$-plane approximation and the periodic symmetry in the study of Zhang et al. (2007) or the limited domain size in Selz and Craig (2015b)].
}

stochastic Plant-Craig convection scheme, global ICON simulations of real cases are used to study upscale error growth induced by the convection scheme up to the planetary scale (Selz 2019). The use of a stochastic convection scheme enables us to represent convective uncertainty and its upscale propagation at relatively low computational cost (Selz and Craig 2015a). Thereby, it is possible to simulate 12 cases each consisting of a 5-member ensemble, in which the only difference between the ensemble members lies in the random seed of the stochastic convection scheme.

We investigate upscale error growth with a PV diagnostic (Teubler and Riemer 2016; Baumgart et al. 2018) that allows us to quantify the relative importance of individual processes to error growth near the tropopause. The partitioning of the processes builds on the PV perspective for midlatitude dynamics and includes the influence of Rossby wave dynamics, baroclinic growth, and moist processes. Additionally, we use a diagnostic for the (phase-filtered) envelope of Rossby waves based on local finite-amplitude wave activity (Ghinassi et al. 2018) to investigate error growth up to the planetary scale.

Based on the PV and envelope diagnostic, we find a distinct sequence of the processes governing upscale error growth: during the first $12 \mathrm{~h}$, error growth is dominated by differences in the convection scheme. This growth is related to differences in the direct PV modification by diabatic processes resulting from their latent heating. Between $12 \mathrm{~h}$ and 2 days, the largest contribution to error growth is related to uppertropospheric divergence, which projects errors from moist processes into the tropopause region. This divergence error growth is associated with vertical-motion errors in regions of mesoscale ascent in the vicinity of cyclones that result from the latent-heating differences. After 2 days, error amplification up to the synoptic scale is dominated by differences in the nonlinear neartropopause dynamics. During this stage, the dynamics of error amplification differ distinctly from the underlying dynamics, which are that of the classical life cycle of moist baroclinic Rossby waves. The envelope diagnostic indicates that a fourth stage of the error growth exists between about day 14.5 and 18 when the predictability time in the PV diagnostic is reached, but when there is still some predictability in the envelope diagnostic. This stage is thus characterized by error growth from the synoptic scale of individual Rossby wave troughs and ridges up to the planetary scale of the (phase-filtered) wave envelope. The processes governing the error evolution during this stage, as identified by the PV diagnostic, are basically indistinguishable from those governing the Rossby wave evolution itself. 
The results of our PV and envelope diagnostic confirm the existence of a multistage behavior of upscale error growth as observed in previous studies (e.g., Zhang et al. 2007; Selz and Craig 2015b; Judt 2018). Importantly, new insight is gained into the processes that govern error growth: By displacing the strong PV gradient associated with the tropopause, upper-level divergence provides an effective way to project errors associated with moist processes directly into the tropopause region. Subsequently, these errors amplify by nonlinear near-tropopause dynamics [the importance of nonlinear dynamics was shown explicitly by Baumgart et al. (2018)]. This notion is distinct from the projection of errors on differences in the release of baroclinic instability, which is in our simulations only of minor importance to the error growth during this stage. Our results suggest an additional fourth stage of planetary-scale error growth that was missing in the conceptual model of Zhang et al. (2007).

Because of the initial-condition and model errors contained in current state-of-the-art operational weather prediction models, one can expect a different errorgrowth behavior in current operational forecasts than in the upscale error-growth simulations investigated here. For instance, the error growth in a recently investigated operational case (Baumgart et al. 2018) did not show the first two stages of upscale error growth that we found in the present study. By contrast, it was dominated by differences in the (balanced) near-tropopause dynamics right from the beginning. For the same data as investigated here, Selz (2019) calculated explicitly that the error level at day 3.5 of our upscale error-growth simulations corresponds to the initial-condition uncertainty of the current ECMWF data assimilation system. Indeed, at day 3.5 upscale error growth in our simulations is already in stage 3 (error growth by near-tropopause dynamics). These results provide evidence that the first two stages do not dominate the error growth in operational forecasts. Nevertheless, stochastic perturbations are necessary to increase the spread in current ensemble forecasting systems that would be underdispersive otherwise. In that sense, upscale error growth is of practical importance to current weather forecasts, albeit not as the dominant mechanism in an average sense. The insight into the processes governing upscale error growth obtained in this study may help to generate stochastic perturbations that effectively initiate an upscale errorgrowth cascade and thereby help to increase spread in current ensemble forecasts.

An improved understanding of upscale error growth may also help to identify regions that are prone to large error growth. As upscale error growth provides a rather effective mechanism to seed an error into the tropopause region within 2 days, one can expect that localized errors along the tropopause establish predominantly in regions with latent heat release below. The dominant contribution of near-tropopause dynamics in the third stage of the error growth suggests that these errors then further amplify along the dynamical tropopause, particularly in regions with a highly distorted tropopause. In that sense, the results of this study point to those regions where one can expect large forecast error growth and thus large forecast uncertainty.

Acknowledgments. The research leading to these results was carried out as part of the Collaborative Research Center SFB/TRR 165 "Waves to Weather" within project A1: "Upscale impact of diabatic processes from convective to near-hemispheric scale" funded by the German Research Foundation (DFG). We thank Franziska Teubler for providing the piecewise PV inversion code and her assistance with the code. We are grateful to Chris Snyder and two anonymous reviewers for constructive comments that helped to improve the presentation of our results.

\section{REFERENCES}

Bauer, P., A. Thorpe, and G. Brunet, 2015: The quiet revolution of numerical weather prediction. Nature, 525, 47-55, https://doi.org/ 10.1038/nature14956.

Baumgart, M., M. Riemer, V. Wirth, F. Teubler, and S. T. K. Lang, 2018: Potential vorticity dynamics of forecast errors: A quantitative case study. Mon. Wea. Rev., 146, 1405-1425, https://doi.org/10.1175/MWR-D-17-0196.1.

Bierdel, L., T. Selz, and G. Craig, 2017: Theoretical aspects of upscale error growth through the mesoscales: An analytical model. Quart. J. Roy. Meteor. Soc., 143, 3048-3059, https://doi.org/ 10.1002/qj.3160.

,-- , and G. C. Craig, 2018: Theoretical aspects of upscale error growth on the mesoscales: Idealized numerical simulations. Quart. J. Roy. Meteor. Soc., 144, 682-694, https://doi.org/ 10.1002/qj.3236.

Buizza, R., and M. Leutbecher, 2015: The forecast skill horizon. Quart. J. Roy. Meteor. Soc., 141, 3366-3382, https://doi.org/ 10.1002/qj.2619.

Davies, H. C., and M. Didone, 2013: Diagnosis and dynamics of forecast error growth. Mon. Wea. Rev., 141, 2483-2501, https:// doi.org/10.1175/MWR-D-12-00242.1.

Davis, C. A., 1992: Piecewise potential vorticity inversion. J. Atmos. Sci., 49, 1397-1411, https://doi.org/10.1175/15200469(1992)049<1397:PPVI>2.0.CO;2.

_ , and K. A. Emanuel, 1991: Potential vorticity diagnostics of cyclogenesis. Mon. Wea. Rev., 119, 1929-1953, https://doi.org/ 10.1175/1520-0493(1991)119<1929:PVDOC >2.0.CO;2.

- M. T. Stoelinga, and Y. H. Kuo, 1993: The integrated effect of condensation in numerical simulations of extratropical cyclogenesis. Mon. Wea. Rev., 121, 2309-2330, https://doi.org/ 10.1175/1520-0493(1993)121<2309:TIEOCI>2.0.CO;2.

Dirren, S., M. Didone, and H. C. Davies, 2003: Diagnosis of "forecast-analysis" differences of a weather prediction system. Geophys. Res. Lett., 30, 2060, https://doi.org/10.1029/ 2003 GL017986. 
Eady, E. T., 1949: Long waves and cyclone waves. Tellus, 1, 33-52, https://doi.org/10.3402/tellusa.v1i3.8507.

Ertel, H., 1942: Ein neuer hydrodynamischer Erhaltungssatz (A new hydrodynamical conservation principle). Meteor. Z., 30 (36), 543-544.

Ghinassi, P., G. Fragkoulidis, and V. Wirth, 2018: Local finiteamplitude wave activity as a diagnostic for Rossby wave packets. Mon. Wea. Rev., 146, 4099-4114, https://doi.org/ 10.1175/MWR-D-18-0068.1.

Heifetz, E., C. H. Bishop, B. J. Hoskins, and J. Methven, 2004: The counter-propagating Rossby-wave perspective on baroclinic instability. I: Mathematical basis. Quart. J. Roy. Meteor. Soc., 130, 211-231, https://doi.org/10.1002/qj.200413059610.

Hohenegger, C., and C. Schär, 2007: Atmospheric predictability at synoptic versus cloud-resolving scales. Bull. Amer. Meteor. Soc., 88, 1783-1793, https://doi.org/10.1175/BAMS-88$11-1783$.

Holton, J. R., and G. J. Hakim, 2012: An Introduction to Dynamic Meteorology. Academic Press, 552 pp.

Hoskins, B. J., M. E. McIntyre, and A. W. Robertson, 1985: On the use and significance of isentropic potential vorticity maps. Quart. J. Roy. Meteor. Soc., 111, 877-946, https://doi.org/ 10.1002/qj.49711147002.

Judt, F., 2018: Insights into atmospheric predictability through global convection-permitting model simulations. J. Atmos. Sci., 75, 1477-1497, https://doi.org/10.1175/JAS-D-17-0343.1.

Liniger, M. A., and H. C. Davies, 2004: Seasonal differences in extratropical potential vorticity variability at tropopause levels. J. Geophys. Res., 109, D17102, https://doi.org/10.1029/ 2004JD004639.

Lorenz, E. N., 1969: The predictability of a flow which possesses many scales of motion. Tellus, 21, 289-307, https://doi.org/ 10.3402/tellusa.v21i3.10086

Lynch, P., 1989: Partitioning the wind in a limited domain. Mon. Wea. Rev., 117, 1492-1500, https://doi.org/10.1175/1520-0493(1989) $117<1492:$ PTWIAL $>2.0 . \mathrm{CO} ; 2$

Nakamura, N., and A. Solomon, 2011: Finite-amplitude wave activity and mean flow adjustments in the atmospheric general circulation. Part II: Analysis in the isentropic coordinate. J. Atmos. Sci., 68, 2783-2799, https://doi.org/10.1175/2011JAS3685.1.

Plant, R. S., and G. C. Craig, 2008: A stochastic parameterization for deep convection based on equilibrium statistics. J. Atmos. Sci., 65, 87-105, https://doi.org/10.1175/2007JAS2263.1.

Quinting, J. F., and S. C. Jones, 2016: On the impact of tropical cyclones on Rossby wave packets: A climatological perspective. Mon. Wea. Rev., 144, 2021-2048, https://doi.org/10.1175/ MWR-D-14-00298.1.

Riemer, M., M. Baumgart, and S. Eiermann, 2014: Cyclogenesis downstream of extratropical transition analyzed by Q-vector partitioning based on flow geometry. J. Atmos. Sci., 71, 4204-4220, https://doi.org/10.1175/JAS-D-14-0023.1.
Rodwell, M. J., and Coauthors, 2013: Characteristics of occasional poor medium-range weather forecasts for Europe. Bull. Amer. Meteor. Soc., 94, 1393-1405, https://doi.org/10.1175/BAMS-D12-00099.1.

— D. S. Richardson, D. B. Parsons, and H. Wernli, 2018: Flowdependent reliability: A path to more skillful ensemble forecasts. Bull. Amer. Meteor. Soc., 99, 1015-1026, https://doi.org/ 10.1175/BAMS-D-17-0027.1.

Saffin, L., J. Methven, and S. L. Gray, 2016: The non-conservation of potential vorticity by a dynamical core compared with the effects of parametrized physical processes. Quart. J. Roy. Meteor. Soc., 142, 1265-1275, https://doi.org/10.1002/qj.2729.

- S. L. Gray, J. Methven, and K. D. Williams, 2017: Processes maintaining tropopause sharpness in numerical models. J. Geophys. Res. Atmos., 122, 9611-9627, https://doi.org/ 10.1002/2017JD026879.

Selz, T., 2019: Estimating the intrinsic limit of predictability using a stochastic convection scheme. J. Atmos. Sci., 76, 757-765, https://doi.org/10.1175/JAS-D-17-0373.1.

— , and G. C. Craig, 2015a: Simulation of upscale error growth with a stochastic convection scheme. Geophys. Res. Lett., $\mathbf{4 2}$, 3056-3062, https://doi.org/10.1002/2015GL063525.

$\longrightarrow$, and $-2015 \mathrm{~b}$ : Upscale error growth in a high-resolution simulation of a summertime weather event over Europe. Mon. Wea. Rev., 143, 813-827, https://doi.org/10.1175/MWR-D-14-00140.1.

Snyder, C., T. M. Hamill, and S. B. Trier, 2003: Linear evolution of error covariances in a quasigeostrophic model. Mon. Wea. Rev., 131, 189-205, https://doi.org/10.1175/1520-0493(2003) $131<0189$ :LEOECI $>2.0$. CO 2 .

Teubler, F., and M. Riemer, 2016: Dynamics of Rossby wave packets in a quantitative potential vorticity-potential temperature framework. J. Atmos. Sci., 73, 1063-1081, https:// doi.org/10.1175/JAS-D-15-0162.1.

Zängl, G., D. Reinert, P. Rípodas, and M. Baldauf, 2015: The ICON (ICOsahedral Non-hydrostatic) modelling framework of DWD and MPI-M: Description of the non-hydrostatic dynamical core. Quart. J. Roy. Meteor. Soc., 141, 563-579, https:// doi.org/10.1002/qj.2378.

Zhang, F., C. Snyder, and R. Rotunno, 2003: Effects of moist convection on mesoscale predictability. J. Atmos. Sci., 60, 1173-1185, https://doi.org/10.1175/1520-0469(2003)060<1173: EOMCOM $>2.0 . \mathrm{CO} ; 2$.

N. Bei, R. Rotunno, C. Snyder, and C. C. Epifanio, 2007: Mesoscale predictability of moist baroclinic waves: Convectionpermitting experiments and multistage error growth dynamics. J. Atmos. Sci., 64, 3579-3594, https://doi.org/10.1175/ JAS4028.1.

Zimin, A. V., I. Szunyogh, D. Patil, B. R. Hunt, and E. Ott, 2003: Extracting envelopes of Rossby wave packets. Mon. Wea. Rev., 131, 1011-1017, https://doi.org/10.1175/1520-0493(2003) 131<1011:EEORWP>2.0.CO;2. 